\title{
CFD BASED DESIGN OF A FILMING INJECTOR FOR N+3 COMBUSTORS
}

\author{
Kumud Ajmani \\ Vantage Partners, LLC \\ Cleveland, Ohio, USA
}

\author{
Hukam Mongia \\ Purdue University \\ West Lafayette, Indiana, USA
}

\author{
Phil Lee \\ Woodward FST, Inc \\ Zeeland, MI, USA
}

\subsection{ABSTRACT}

An effort was undertaken to perform CFD analysis of fluid flow in Lean-Direct Injection (LDI) combustors with axial swirl-venturi elements for next-generation LDI-3 combustor design. The National Combustion Code (NCC) was used to perform non-reacting and two-phase reacting flow computations for a newly-designed pre-filming type fuel injector LDI-3 injector, in a single-injector and a five-injector array configuration. All computations were performed with a consistent approach of mesh-optimization, spraymodeling, ignition and kinetics-modeling. Computational predictions of the aerodynamics of the singleinjector were used to arrive at an optimized main-injector design that meets effective area and fuel-air mixing criteria. Emissions (EINOx) characteristics were predicted for a medium-power engine cycle condition, and will be compared with data when it is made available from experimental measurements. The use of a PDF-like turbulence-chemistry interaction model with NCC's Time-Filtered Navier-Stokes (TFNS) solver is shown to produce a significant impact on the CFD results, when compared with a laminarchemistry TFNS approach for the five-injector computations.

\subsection{INTRODUCTION}

Recent interest within the aviation industry in reducing emissions targets for NOx, CO and UHCs, combined with NASA's N+3 program, have continued the further development of LDI injection concepts. The attendant low emissions levels achieved with these injectors in previous efforts (e.g. the N+2 ERA program) serve as motivation to refine the N+2 designs to obtain even lower emissions, better operability, and flexible-fuel capability. The results of the previous generation (LDI-1) efforts were summarized by [Lee 2007] and [Tacina 2008], and some highlights of some improved (LDI-2) efforts have been reported by [Lee 2013], [Suder 2013] and [Tacina 2014].

Experimental measurements of axial-swirler LDI-2 configurations based on a combination of thirteen simplex-type pressure-atomizing injectors and airblast injectors as designed by Woodward FST (WFST) were performed at WFST (for low P3) and NASA Glenn Research Center (for high P3). The LDI-2 design used the lessons learnt from experimental studies of nine-element LDI-1 arrays with axial-swirlers, as performed and reported by [Tacina 2005]. In more recent work, [Tacina 2014a] and [Hicks 2014] have reported optical measurements of unsteady effects and comparison of cold and reacting flows, respectively, of LDI-1 multipoint configurations with bladed axial-swirlers and simplex injectors.

The National Combustion Code (NCC) has been developed at NASA Glenn Research Center (GRC) through extensive validation with available LDI data, making it very attractive as a tool to help guide technology development of the $2^{\text {nd }}$ generation Lean Direct Injection, LDI-2. Previous work of [Ajmani 2013] and [Mongia, 2008] have shown the importance of establishing CFD best practices for correlating emissions data from state of the art combustions systems.

This paper provides an overview of the efforts undertaken with the NCC to use the lessons learned in LDI-2 injector design, to perform a detailed CFD analysis of two different LDI-3 configurations. The goal is to use CFD to optimize the LDI-3 injector design, before final design hardware is machined and tested. The CFD is also used to compute heat release and NOx emissions for a newly designed pre-filming fuel injector LDI-3 design proposed by Woodward FST, Inc. The current re-design follows the recommendations from the CFD analysis performed from the initial LDI3 injector design, which had 
several deficiencies in providing adequate fuel-air mixing [Ref JPC 2015 Ajmani]. The CFD results presented here for a five-element injection module based on an NCC-optimized single-element injector design, will eventually be compared with experimental measurements to be obtained in testing of the LDI-3 design at NASA GRC, and Woodward FST, Inc, at various engine power conditions.

\subsection{COMPUTATIONAL APPROACH WITH THE NCC}

The National Combustion Code (NCC) was used to perform simulations of several Woodward, FST, Inc., LDI-3 injector configurations. The NCC is a state-of-the-art computational tool that is capable of solving the time-dependent, Navier-Stokes equations with chemical reactions. The NCC is being developed primarily at the NASA Glenn in order to support combustion simulations for a wide range of applications, and has been extensively validated and tested for low-speed chemically reacting flows.

The NCC uses second-order accurate central-differences for the convective and diffusion flux discretization, and a Jameson operator (a blend of 2nd and 4th-order dissipation terms) for numerical stability. The second and fourth order dissipation parameters are typically set to $10^{-4}$ and 0.05 , respectively ([Swanson 1997]). The value of $\mathrm{k}_{2}$, the constant that scales the second order dissipation gradient switch, is typically set to 0.25 . In order to enhance convergence acceleration in pseudo-time, implicit residual smoothing is used to smooth the computed residuals in NCC RANS. Turbulence closure is obtained by using a two-equation, cubic $\mathrm{k}-\varepsilon$ model with variable $\mathrm{C} \mu$ ([Shih 1998]) and dynamic wall functions with pressure gradient effects ([Shih 2000]).

\subsection{LDI-3 GEOMETRY, MESHING AND CFD SETUP}

In order to overcome the fuel-air mixing deficiencies of the first LDI-3 injector design reported earlier [Ajmani 2015], a second injector design incorporating a "filming injection module" was formulated and computationally analyzed. NCC was used to perform simulations of several multiple element modules of an LDI-3 injector array proposed by Woodward FST, Inc (WFST). Each injection module consists of a combination central pilot-injector and four (or six) surrounding main-injection elements (see figure 1). A candidate LDI-3 arrangement with an assembly of three modules with seven, five and seven injectors respectively, for a total of seventeen injectors, was designated as the 'baseline' configuration. The CFD-led injector optimization study reported in this paper using NCC focuses on the 'central' five-injector module. A separate NCC CFD study of the seven-injector module, and the complete seventeen-injector array, will be reported in later publications.

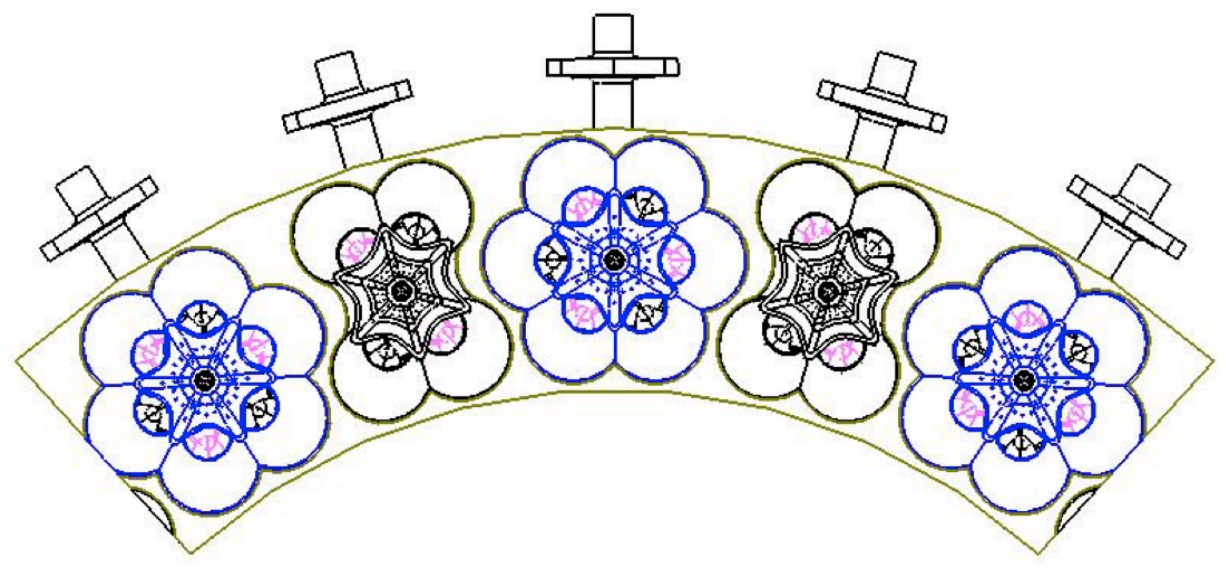

Figure 1. Dome-layout with alternating seven- and five-injector modules for an LDI-3 injector array

The five-injector module of the seventeen-injector LDI-3 array as supplied by WFST (see figure 1) was imported into the CUBIT mesh-generation software, to create a fully tetrahedral mesh with approximately 10M elements. ). Best-practices for meshing of LDI-1 injector elements reported in [Ajmani, 
2013 (ASM)] were leveraged for LDI-3 meshing. Each blade passage of the axial-bladed airflow swirlers was meshed as an individual block, and these blocks were then "imprinted" and merged with upstream and downstream connecting volumes at their respective common surfaces. This modular approach to meshing ensured (a) fine control of meshing in critical areas, (b) consistency of meshing across similar geometric elements, and (c) allowed for 'drop-in' replacement of the axial air swirlers for the main injectors, and/or the radial air-swirler for the pilot, without needing to regenerate the complete mesh for the entire configuration.

\subsection{CFD ANALYSIS OF SINGLE INJECTOR WITH NCC}

\subsection{LIQUID-FILMING MAIN-INJECTION MODULE - SINGLE INJECTOR}

A detailed analysis of one of the four main injectors was the first step in the parametric design of the five-injector module described in this paper. Recall that the five-injector module consists of four main injectors with filming fuel injection, surrounding a central pilot fed by a simplex fuel injector. The geometry and surface-mesh detail of one of four the main injectors for a "filming injector" configuration is shown in figure 2 . A fully tetrahedral mesh consists of approximately $4 \mathrm{M}$ elements was generated with the CUBIT software, following the mesh-resolution criteria for individual components detailed in section 3 . The goal of performing the non-reacting flow analysis for the three configurations was to determine the optimal configuration that would (a) maximize the total $\mathrm{AC}_{\mathrm{d}}$ of the main injector and (b) minimize the near-wall flow separation downstream of the throat section

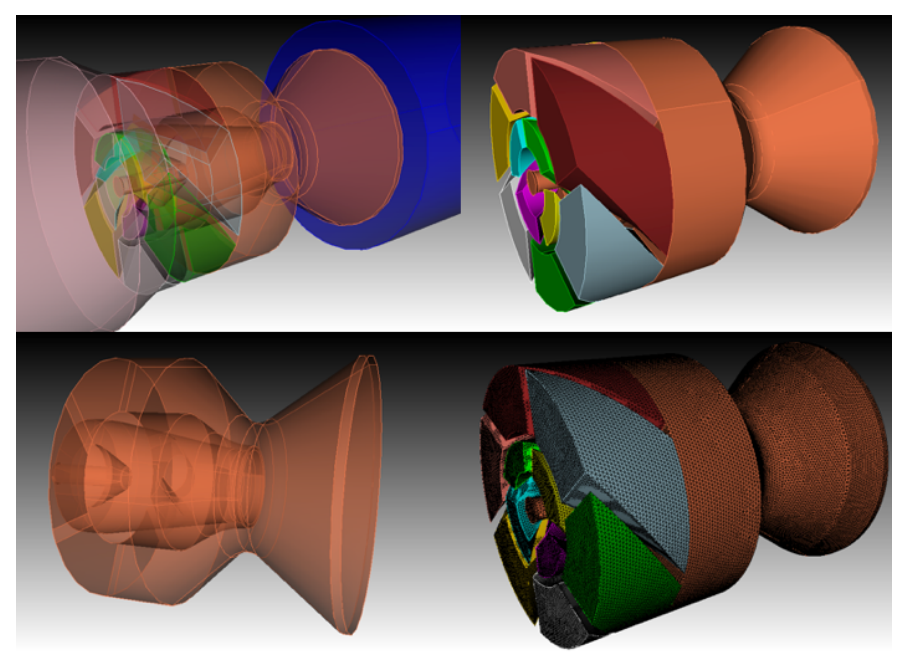

Figure 2. Filming Injector geometry and surface mesh for co-rotating inner/outer swirler configuration

The boundary conditions for the CFD analysis were:

- Fixed total-pressure $\left(\mathrm{P}_{3}=101 \mathrm{kPa}\right)$ and total-temperature $\left(\mathrm{T}_{3}=295 \mathrm{~K}\right)$ at inflow boundaries

- Fixed static-pressure $\left(\mathrm{P}_{3}-\Delta \mathrm{P}\right)$ at outflow $\left(\Delta \mathrm{P}=1 \%\right.$ of $\left.\mathrm{P}_{3}\right)$

- Adiabatic, no-slip conditions at all walls

Non-reacting flow analysis with RANS and TFNS/VLES was first performed for three different swirler configurations. Each configuration consisted of a combination of co-axial inner and outer axiallybladed swirler elements. The inner and outer rows of swirlers have four and six bladed passages, respectively. In addition to the two rows of swirlers, an extra airflow passage is also created along the injector centerline, to produce a relatively high-momentum jet at the injector core. Three variations of the liquid-filming injector were studied, where the angle of the outer swirlers and the relative orientation of the inner and outer swirler passages were varied:

1. outer/inner swirlers at $52^{\circ} / 60^{\circ}$ (co-rotating)

2. outer/inner swirlers at $52^{\circ} / 60^{\circ}$ (counter-rotating)

3. outer/inner swirlers at $48^{\circ} / 60^{\circ}$ (counter-rotating) 
Each non-reacting flow simulation consisted of NCC RANS computations till a 'mass-imbalance (outflow-inflow) convergence of $0.1 \%$ over 500 consecutive NCC RANS iterations was achieved. For a typical case, 100,000 RANS iterations at a CFL of 1.95 were run, to obtain a converged, steady-state, nonreacting flowfield. The CFD computed mass-flow rate at the inlet of each injector was used to determine the $\mathrm{AC}_{\mathrm{d}}$ of each individual injector, and the total $\mathrm{AC}_{\mathrm{d}}$ of each configuration was compared to measured values of $\mathrm{AC}_{\mathrm{d}}$ provided by Woodward FST, Inc.

\subsection{Non-Reacting Flow CFD Results: Co-Rotating Swirlers vs. Counter-Rotating Swirlers}

\begin{tabular}{|c|c|c|c|}
\hline Swirler Configuration & Measured $\left(\right.$ in $\left.^{2}\right)$ & Computed $\left._{\left(i^{2}\right.}{ }^{2}\right)$ & Error (\%) \\
\hline $52^{\circ} / 60^{\circ}(\mathrm{OAS} / \mathrm{IAS})$ co-rotating & 0.137 & 0.1411 & 3.0 \\
\hline $52^{\circ} / 60^{\circ}(\mathrm{OAS} / \mathrm{IAS})$ counter-rotating & 0.134 & 0.1259 & -1.1 \\
\hline $48^{\circ} / 60^{\circ}(\mathrm{OAS} / \mathrm{IAS})$ counter-rotating & 0.144 & 0.1467 & 1.9 \\
\hline
\end{tabular}

Table 1. Effect of outer/inner air swirler arrangement on effective area $\left(\mathrm{AC}_{\mathrm{d}}\right)$
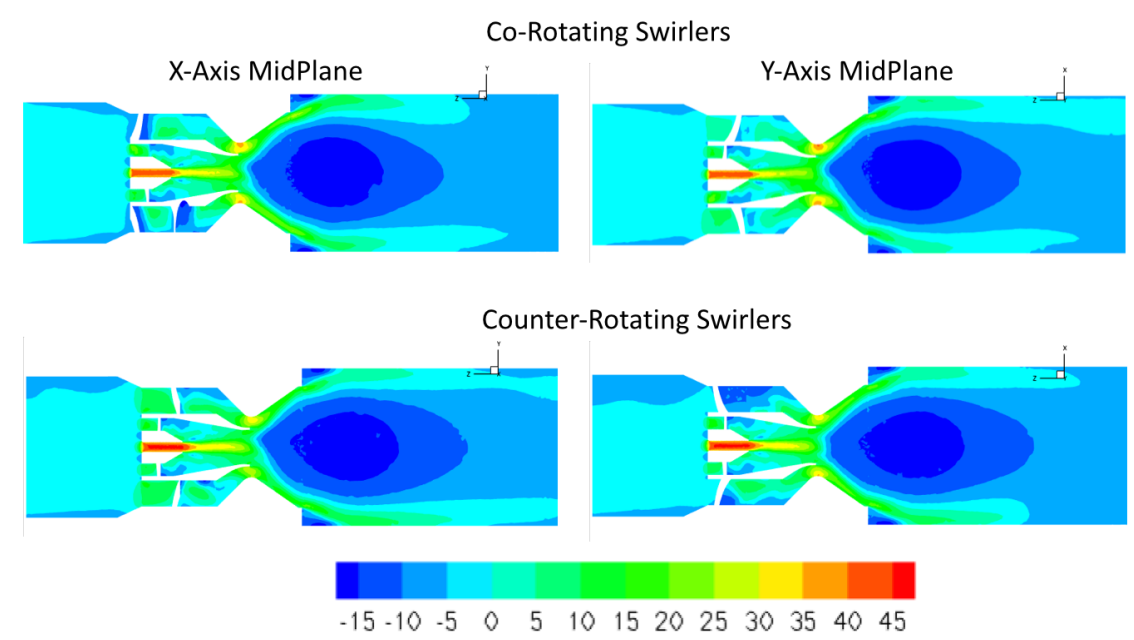

Figure 3. NCC RANS axial-velocity ( $\mathrm{m} / \mathrm{s}$ ) contours for co-rotating (top) and counter-rotating (bottom) swirlers ( $52^{\circ}$ outer, $60^{\circ}$ inner air swirlers); non-reacting flow at $\mathrm{P}_{3}=1 \mathrm{~atm}, \mathrm{~T}_{3}=295 \mathrm{~K}, \Delta \mathrm{P}=1 \%$

The counter-rotating configuration has very similar $\mathrm{AC}_{\mathrm{d}}$ to co-rotating, and the flow patterns seem very similar. The counter-rotating configuration is selected for better uniformity of velocity profile at throat and a slightly more compact central recirculation zone downstream of the throat. Finally, the configuration with $48^{\circ}$ OAS is chosen over the $52^{\circ} \mathrm{OAS}$, as this yields a $7.5 \%$ increase in $\mathrm{AC}_{\mathrm{d}}$ for the injector. The CFD predictions of $\mathrm{AC}_{\mathrm{d}}$ for all three configurations are within $3 \%$ error of the measured $\mathrm{AC}_{\mathrm{d}}$.

\subsection{Reacting Flow CFD Results: Counter-Rotating 48/60 Swirlers}

The optimal configuration of $48^{\circ} / 60^{\circ}$ counter-rotating OAS/IAS identified with non-reacting flow NCC RANS, was further analyzed with reacting flow NCC TFNS/VLES [Ref]. This analysis was performed to determine the reacting flow characteristics and NOx emissions performance of the current LDI-3 single injector, and compare it to previous generation LDI-2 injectors reported in [Ref]. The current injector was analyzed for a 'medium' power engine cycle condition represented by $\mathrm{P}_{3}=130 \mathrm{psi}, 4 \% \Delta \mathrm{P}$, $\mathrm{T}_{3}=1000 \mathrm{~F}(811 \mathrm{~K})$ and $\mathrm{FAR}=0.0214$. Figures 3 and 4 show some of the flow-field characteristics and liquiddroplet distribution in the proposed LDI-3 injector. The contours are plotted from mean values of the final 3100 physical time-steps of the TFNS/VLES solution, which represents 1.5 flow-through cycles for the injector.

The axial-velocity contours show that the length of the primary recirculation zone is approximately 
$2 \mathrm{D}$, where $\mathrm{D}$ is exit diameter of the venturi. Further optimization of the swirlers may be necessary in order to reduce the length of this primary recirculation zone by $25-50 \%$. The predicted 'mass-weighted areaaveraged' exit temperature for the injector $\left(\mathrm{T}_{4}\right)$ is $1595 \mathrm{~K}$, which is $3 \%$ higher than an 'ideal' equilibrium temperature of $1545 \mathrm{~K}$ (for $\mathrm{T}_{3}=811 \mathrm{~K}, \mathrm{FAR}=0.0214$ ). The temperature contours show locally high temperature streaks in the vicinity of the walls of the diverging section of the venturi. This may necessitate the addition of cooling air holes in venturi walls. The predicted 'mass-weighted area-averaged' exit EINOx for the injector is 9.5 , which is approximately $25 \%$ higher than the corresponding EINOx for an equivalent LDI-2 injector. Mitigation of the near-wall high-temperature streaks by addition of wall-cooling holes, and reduction of the swirl angle of the outer swirlers are two strategies that could help reduce EINOx emissions.
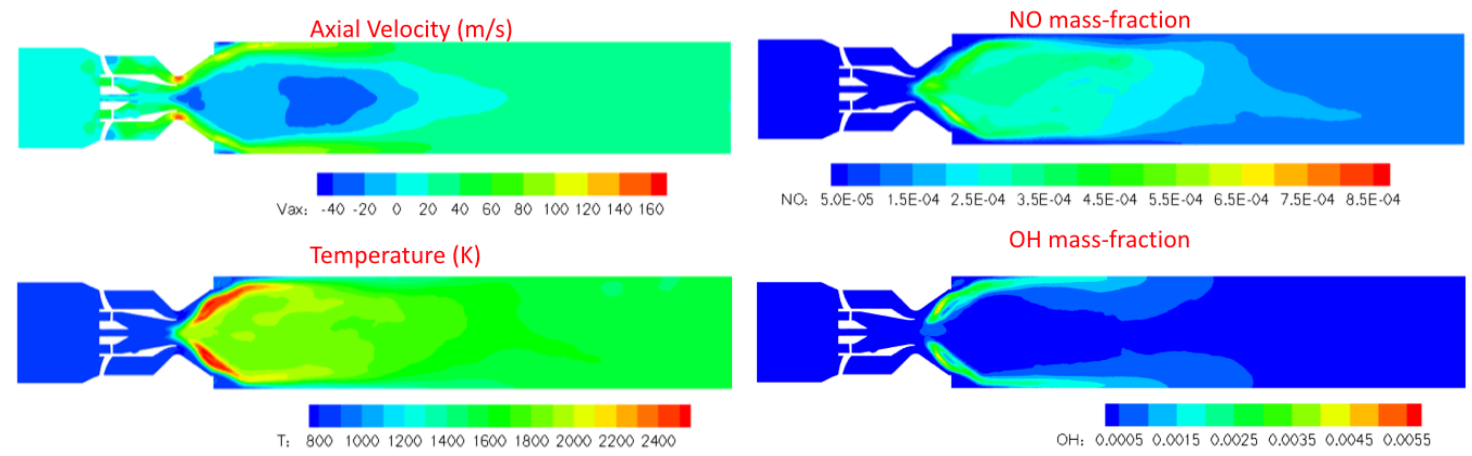

Figure 3. Left: Axial velocity (top) and Temperature (bottom). Right: NO mass-fraction (top) and $\mathrm{OH}$ mass-fraction (bottom). Time-averaged TFNS/VLES reacting solution.

Note that the fuel is injected through a slot that is tangential to the surface of the inner air passage of the injector, downstream of the inner air swirlers. The design intent is that the swirling air will transport the fuel downstream, and that the liquid film will be sheared into droplets as the inner and outer air streams meet at the throat section. For modeling purposes, the fuel is injected at an axial location immediately downstream of the pre-filmer surface. Sixteen discrete fuel streams spread evenly along the prefilmer circumference are used to simulate the breakup of the fuel-film. A pre-defined droplet size SMD of 8 microns, and a very low injection velocity of $5 \mathrm{~m} / \mathrm{s}$ to model the annular film exiting the pre-filmer, is used in the Lagrangian modeling [Ref Suri] of the fuel particles. Details of the predicted droplet distribution of the newly designed liquid filming injector are shown in figure 4. Most of the liquid fuel has evaporated immediately downstream of the dump plane, and the isometric view shows a fairly even distribution of fuel in the circumferential direction. These characteristics of the newly designed pre-filmer for the current design iteration largely overcome the fuel-air mixing deficiencies of the previous parametric design reported in [Ref JPC 2015].
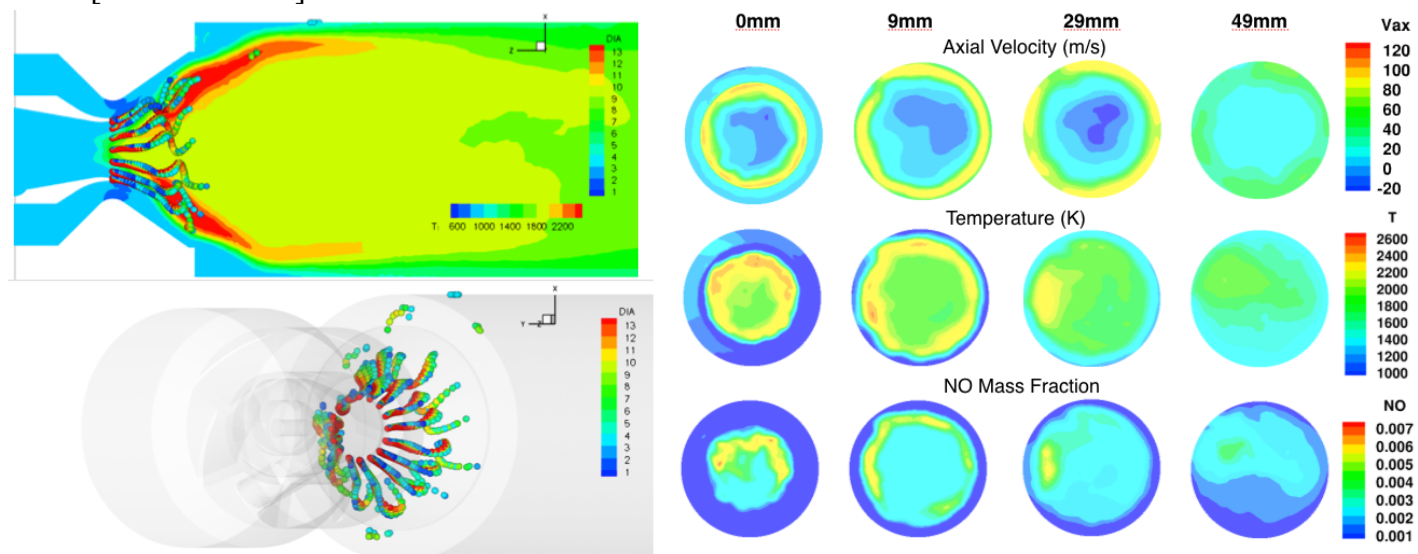

Figure 4. Left: Temperature $(\mathrm{K})$ contours and predicted particle distributions. Right: Cross-sectional contours of flow quantities at dump plane. Time-averaged TFNS/VLES reacting-flow solution. 


\subsection{SUMMARY OF COMPUTATIONS WITH THE NCC - FIVE INJECTOR CONFIGURATION}

The newly-designed pre-filming injector (see section 4) was integrated into a five-injector configuration representing the central five-injector module of a nineteen-injector flame-tube assembly (see section 3). A detailed computational study of the integrated five-injector module is reported in this section of the paper. Some design features of this five-element injector module (see figure 5) are given below (see figure 5).

- Integrated "five-element" injector module consists of one central 'Pilot' injector and four adjacent 'Main' injectors

- Four Main injectors with $48^{\circ} / 60^{\circ}$ axial air swirlers (see section 4) followed by convergingdiverging venturi; pre-filming fuel injection (filmer chamber exit diameter $=0.24$ ")

- Central Pilot injector with multiple, radial inflow slots for airflow. Air inflow direction is 51\% offset with respect to the injector centerline. The injection element is fed by a standard pressureatomizing, axial fuel injector.

A tetrahedral mesh with $10 \mathrm{M}$ elements (see figure 5) was generated for the five-element module, using the mesh-generation methodology described in section 3 .
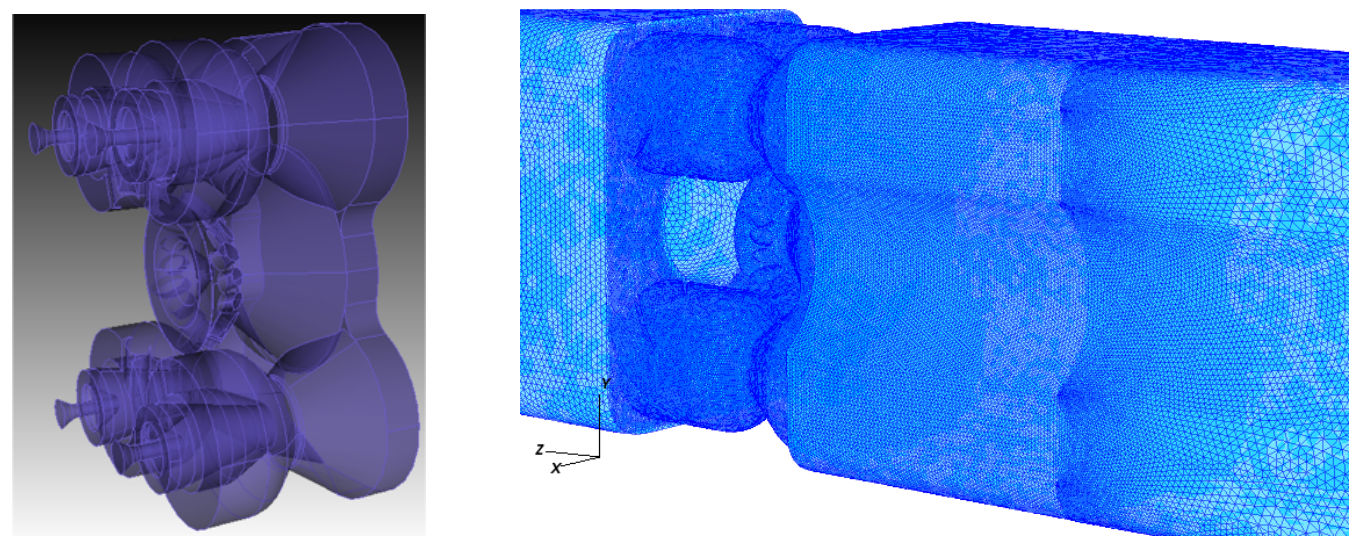

Figure 5. Filming Injector geometry and surface mesh for co-rotating five-element configuration

\begin{tabular}{|c|c|c|c|}
\hline Injector Element & Venturi L (in) & Venturi Dia (in) & Venturi Angle (\%) \\
\hline PILOT 51\% Offset PILOT & 0.425 & 1.3 & 43.3 \\
\hline MAIN $48^{\circ} / 60^{\circ}$ OAS/IAS & 0.35 & 1.0 & 35.5 \\
\hline
\end{tabular}

Table 2.

Geometry

details of a five-element injection module created with a central pilot, four mains

A comparison of computational results for non-reaction flow solutions from NCC RANS and NCC TFNS/VLES are shown in figures 6 and 7, respectively. Note that the RANS solution is plotted after 55000 iterations at $\mathrm{CFL}=1.95$, and the TFNS/VLES solution represents a time-average of 10000 time-steps (1.e6s). The flow conditions evaluated are identical to the single-element injector computations reported in section 4, i.e. a 'medium' power engine cycle condition represented by $\mathrm{P}_{3}=130 \mathrm{psi}, 4 \% \Delta \mathrm{P}, \mathrm{T}_{3}=1000 \mathrm{~F}$ $(811 \mathrm{~K})$ and $\mathrm{FAR}=0.0214$. The axial-velocity contours in figure 6 show that the largest differences between NCC RANS and NCC TFNS/VLES are in the prediction of the central recirculation zone (CRZ) behind the central pilot injector. The RANS solution shows almost no CRZ, and two very large corner recirculation zones (CoRZ) attached to the diverging surface of the pilot venturi. The TFNS solution shows a very well defined, strong CRZ and a much smaller CoRZ behind the pilot. The flow characteristics of axial velocity behind the four main injectors are very similar, for both the RANS and TFNS/VLES predictions with NCC. 
Axial-velocity contours at six locations downstream of the injector-module assembly's dump plane are shown in figure 7. The differences between RANS and TFNS/VLES predictions for the pilot element airflow are clearly delineated in the comparison plots at all six locations. The TFNS/VLES solutions show good symmetry, whereas the RANS predictions become increasingly asymmetric with downstream distance. The diagonal line drawn in the $40 \mathrm{~mm}$ plot for the TFNS/VLES solution represents the antisymmetric effect of clockwise (top right, bottom left) and counter-clockwise (top left, bottom right) orientation of the air-swirlers of the main injection elements. Interaction between the respective flow-fields behind the pilot and the four surrounding main elements is indicated by the TFNS/VLES solution. While there is no experimental data available yet for this five-element module, the symmetric nature of the TFNS/VLES solutions indicates a more 'realistic' aerodynamic prediction for this configuration.

RANS
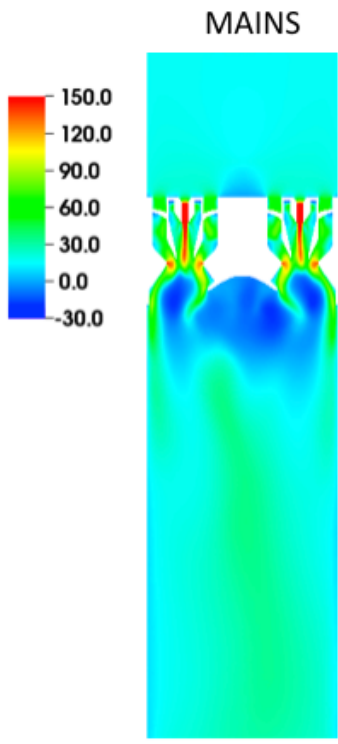

PILOT
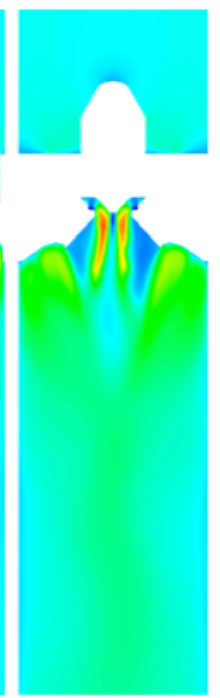

MAINS

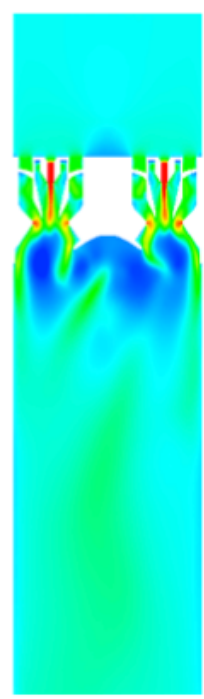

TFNS

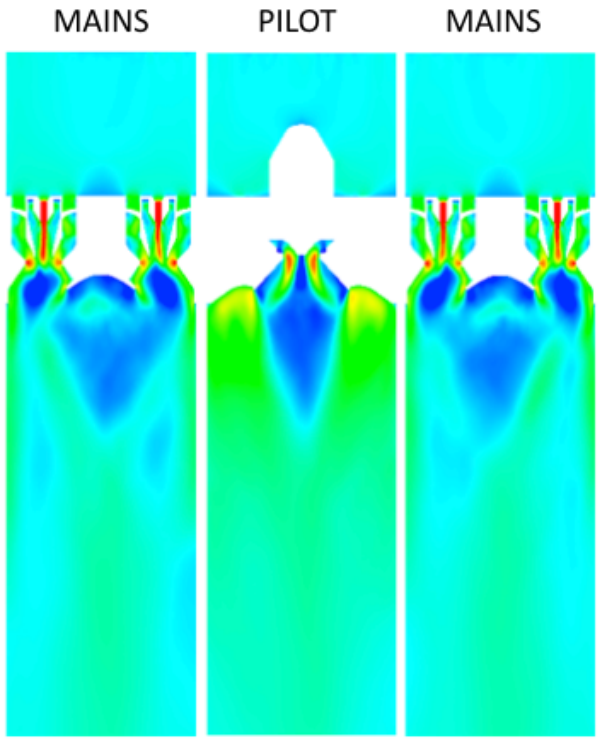

Figure 6. Axial velocity $(\mathrm{m} / \mathrm{s})$ contours in three axial cross-sections through centerline of two main injectors and the pilot injector. Non-Reacting Flow. Left: RANS solution. Right: TFNS/VLES solution

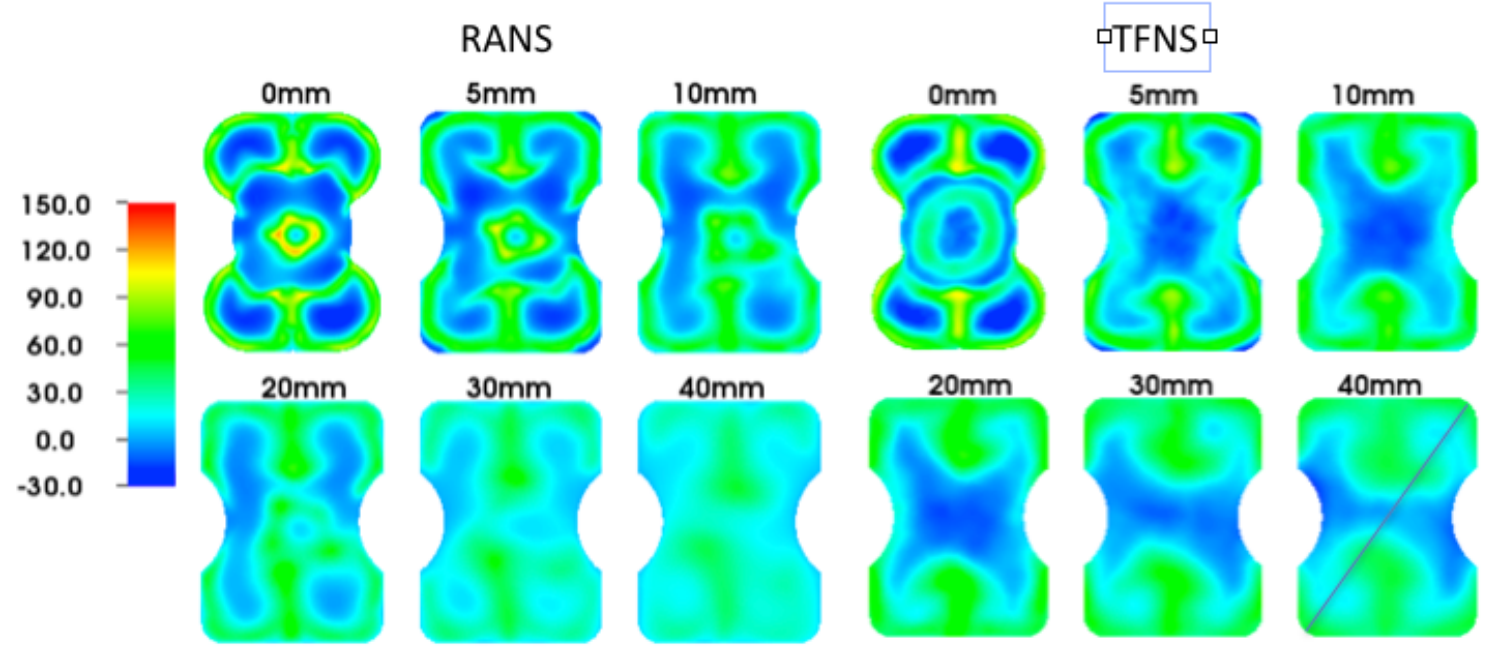

Figure 7. Axial velocity $(\mathrm{m} / \mathrm{s})$ contours in six cross-sections at various axial locations downstream of dump plane located at 0mm. Non-Reacting Flow. Left: RANS solution. Right: TFNS/VLES solution

Iso-surfaces of zero axial-velocity representing the boundary between forward and reverse axialvelocity are shown in figure 8. A very distinct core of reverse flow is shown in the TFNS/VLES predictions for the pilot element, whereas the RANS solution shows no such flow feature. This three-dimensional view indicates the existence of very strong vortex core behind the central pilot injector element. 
RANS

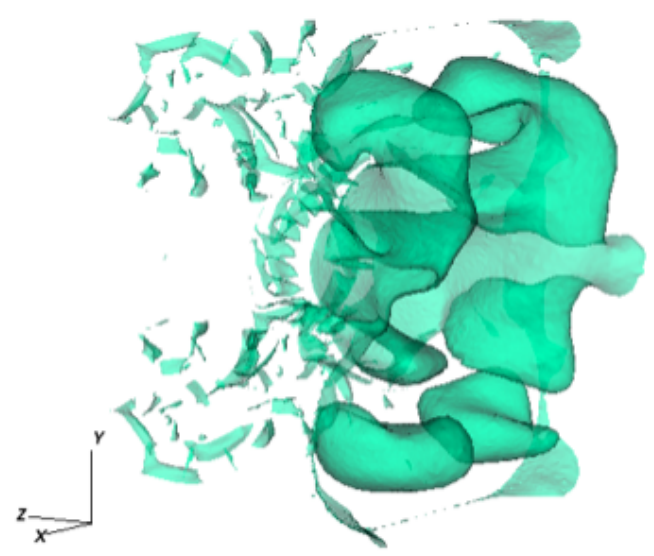

TFNS

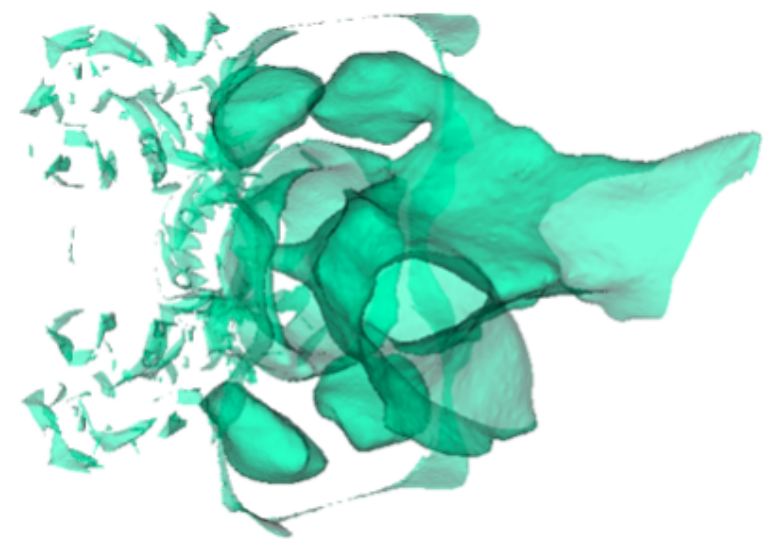

Figure 8. Iso surfaces of zero axial velocity $(\mathrm{m} / \mathrm{s})$ from non-reacting flow CFD analysis. Left: RANS solution. Right: TFNS/VLES solution

A comparison of experimentally measured effective area $\left(\mathrm{AC}_{\mathrm{d}}\right)$ and $\mathrm{NCC}$ predictions with RANS and TFNS/VLES for the five-element module is shown in Table 3. The NCC RANS prediction of total effective area is within $3 \%$ of the total measured $\mathrm{AC}_{\mathrm{d}}$, while the NCC TFNS prediction for $\mathrm{AC}_{\mathrm{d}}$ is $10 \%$ higher than the measured value. Note that the NCC TFNS values for $\mathrm{AC}_{\mathrm{d}}$ were computed from a time-averaged TFNS solution over 10,000 time-steps, and $\mathrm{AC}_{\mathrm{d}}$ predictions for both NCC RANS and NCC TFNS are within 1\% error of each other.

\begin{tabular}{|c|c|c|c|c|}
\hline Method & Total $\left(\mathrm{in}^{2}\right)$ & Mains $\left(\right.$ in $\left.^{2}\right)$ & Pilot (in $\left.{ }^{2}\right)$ & Error (\%) \\
\hline Measured & 0.720 & 0.575 & 0.145 & \\
\hline NCC RANS & 0.744 & 0.620 & 0.124 & 3.3 \\
\hline NCC TFNS & 0.752 & 0.621 & 0.131 & 4.4 \\
\hline
\end{tabular}

Table 3. Effective area $\left(\mathrm{AC}_{\mathrm{d}}\right)$ predictions for the five-element injector module

\subsection{FIVE INJECTOR MODULE - SWIRLER OPTIMIZATION}

In order to attempt to mitigate some of the near wall high-temperature effects observed in the singleinjector analysis (see section 4.3), the outer air-swirler turning angle was decreased from $48^{\circ}$ to $45^{\circ}$, to produce an air-swirler designated as $45^{\circ} / 60^{\circ}$ OAS/IAS. This change also has the added benefit of marginally increasing the predicted $\mathrm{AC}_{\mathrm{d}}$ of the main injector by $3 \%$. Figures 9 and 10 show axial-velocity comparisons between the $48^{\circ} / 60^{\circ}$ and $45^{\circ} / 60^{\circ} \mathrm{OAS} / \mathrm{IAS}$ swirlers, respectively. A decrease in the OAS angle destroys the prominent central recirculation zone (CRZ) of the $48^{\circ} / 60^{\circ}$ swirlers. The CRZ is replaced with corner recirculation zones (CoRZ) near the inner walls of the venturis and the main walls of the injector for the $45^{\circ} / 60^{\circ}$ OAS/IAS swirlers (figure 9 , right). The decreased OAS angle of $45^{\circ}$ also has a marginal effect on decreasing the size of the CoRZ behind the pilot injector.

Contours of axial-velocity at six different axial locations (figure 10) show the substantial effect of the decreasing the OAS angle from $48^{\circ}$ to $45^{\circ}$. The transition from strong CRZ (48/60 OAS/IAS case) aerodynamics to a strong CoRZ airflow pattern is clearly seen in the comparison plots, particularly at the $0 \mathrm{~mm}, 10 \mathrm{~mm}$, and $20 \mathrm{~mm}$ locations in figure 10 . The NCC RANS comparisons indicates that the $45^{\circ}$ OAS configuration is less stable from an operability standpoint (presence of CRZ is preferred for better operability). In order to improve the overall operability, a final design iteration was performed by increasing the amount of swirl in the pilot swirlers for the $45^{\circ} / 60^{\circ}$ OAS/IAS main swirler configuration. 


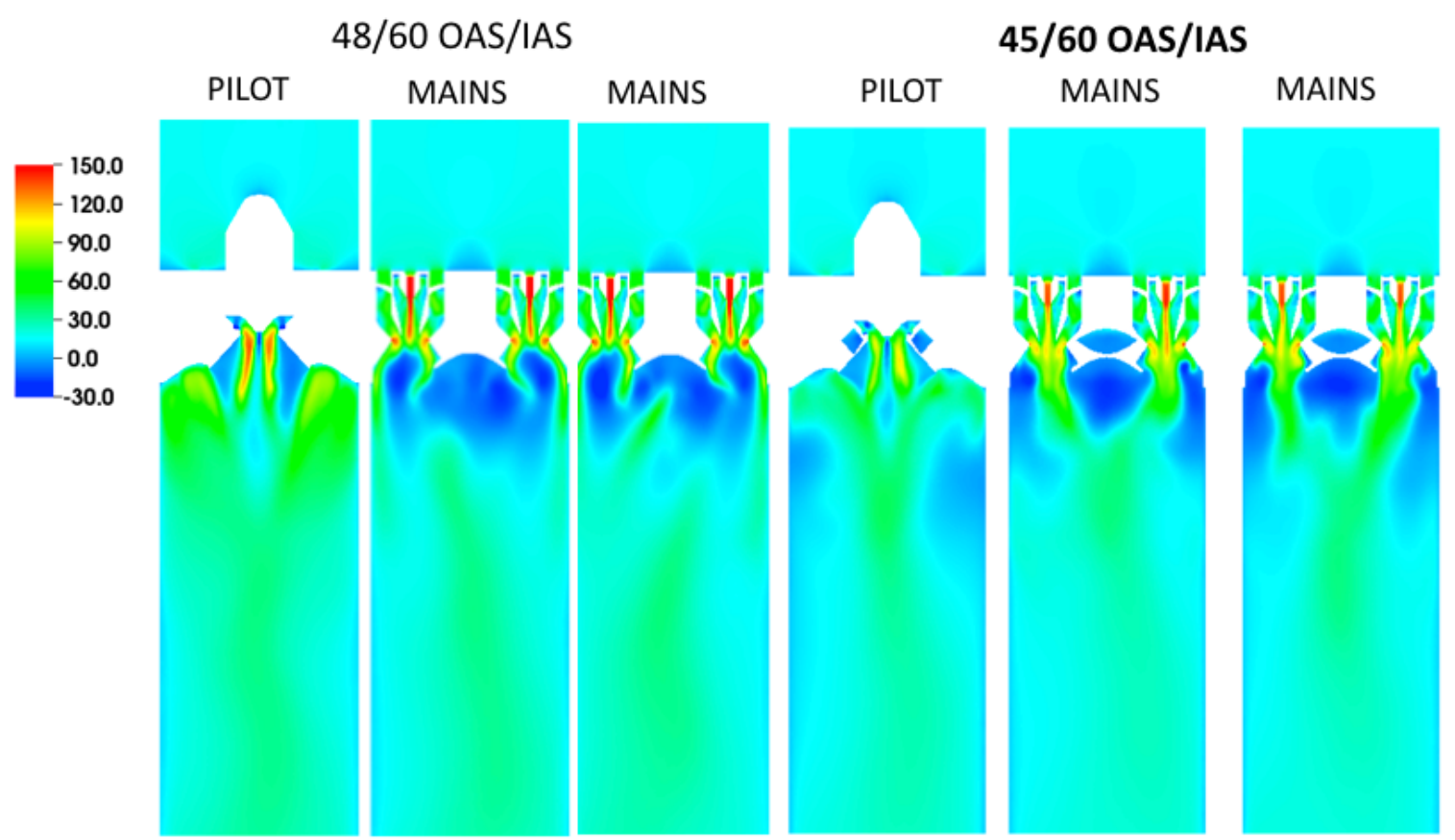

Figure 9. Axial velocity $(\mathrm{m} / \mathrm{s})$ contours in three axial cross-sections through centerline of two main injectors and the pilot injector. Left: $48^{\circ} / 60^{\circ}$ OAS/IAS Main Swirlers. Right: $\mathbf{4 5}^{\circ} / 60^{\circ}$ OAS/IAS Swirlers

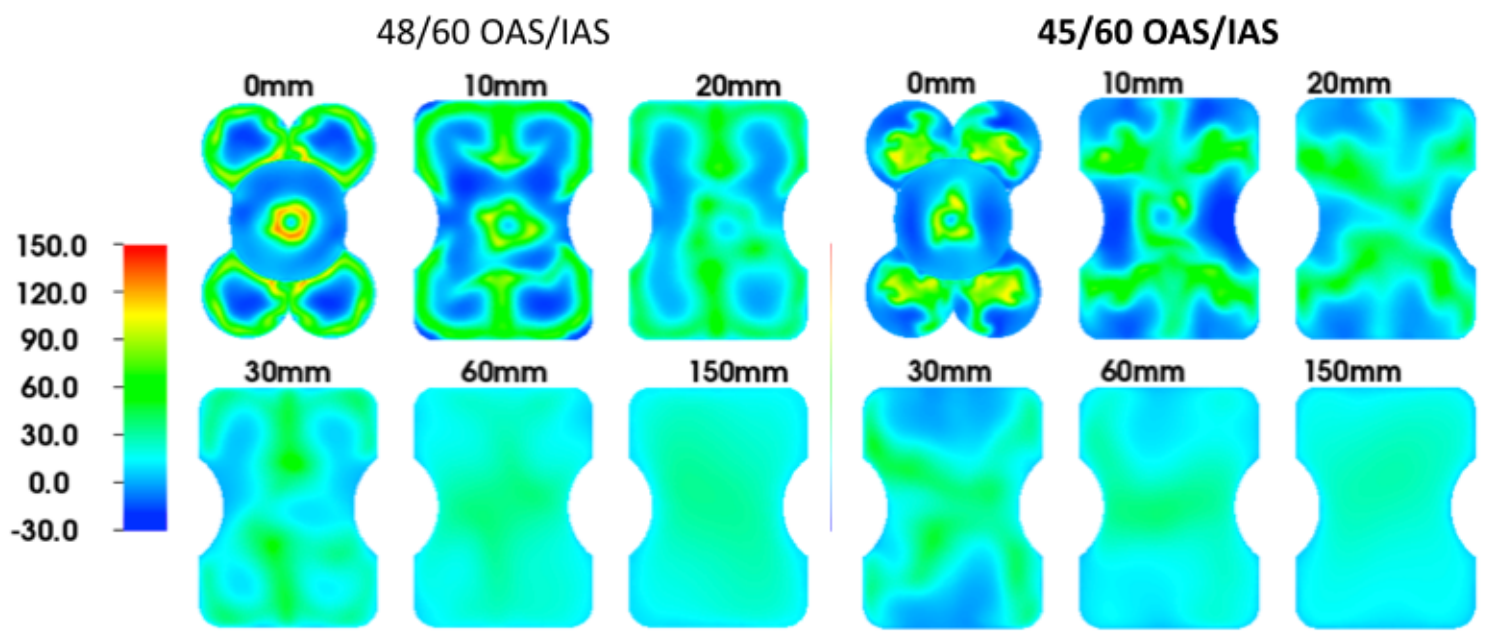

Figure 10. Axial velocity $(\mathrm{m} / \mathrm{s})$ contours in six cross-sections at various axial locations downstream of dump plane located at $0 \mathrm{~mm}$. Left: $48^{\circ} / 60^{\circ} \mathrm{OAS} / \mathrm{IAS}$ Main Swirlers. Right: $\mathbf{4 5}^{\circ} / 60^{\circ} \mathrm{OAS} / \mathrm{IAS}$ Swirlers

Figure 11 shows a comparison of contours of axial-velocity for a 'high-swirl' pilot injector, where the radial inflow holes are now $63 \%$ offset from the centerline, as compared to $51 \%$ offset for the previous 'low-swirl pilot. In addition, all the radial inflow holes are of equal size, as compared to the larger alternating holes of the 'low swirl' pilot. The flow behind the 'high-swirl' pilot shows the presence of a strong CRZ, as compared to weak CoRZ regions behind the 'low-swirl' pilot. In addition, the 'high-swirl' pilot configuration manages to re-establish the CRZ behind the main injectors,. Recall that the CRZ features were originally present in the $48^{\circ} / 60^{\circ}$ OAS/IAS main swirler geometry, but had been replaced by the CoRZ flow features of the $45^{\circ} / 60^{\circ}$ OAS/IAS mains (figure 11, right). This final combination of 'highswirl' pilot swirler and $45^{\circ} / 60^{\circ} \mathrm{OAS} / \mathrm{IAS}$ main swirlers was selected for fabrication and also detailed reacting-flow analysis reported in the next section. 


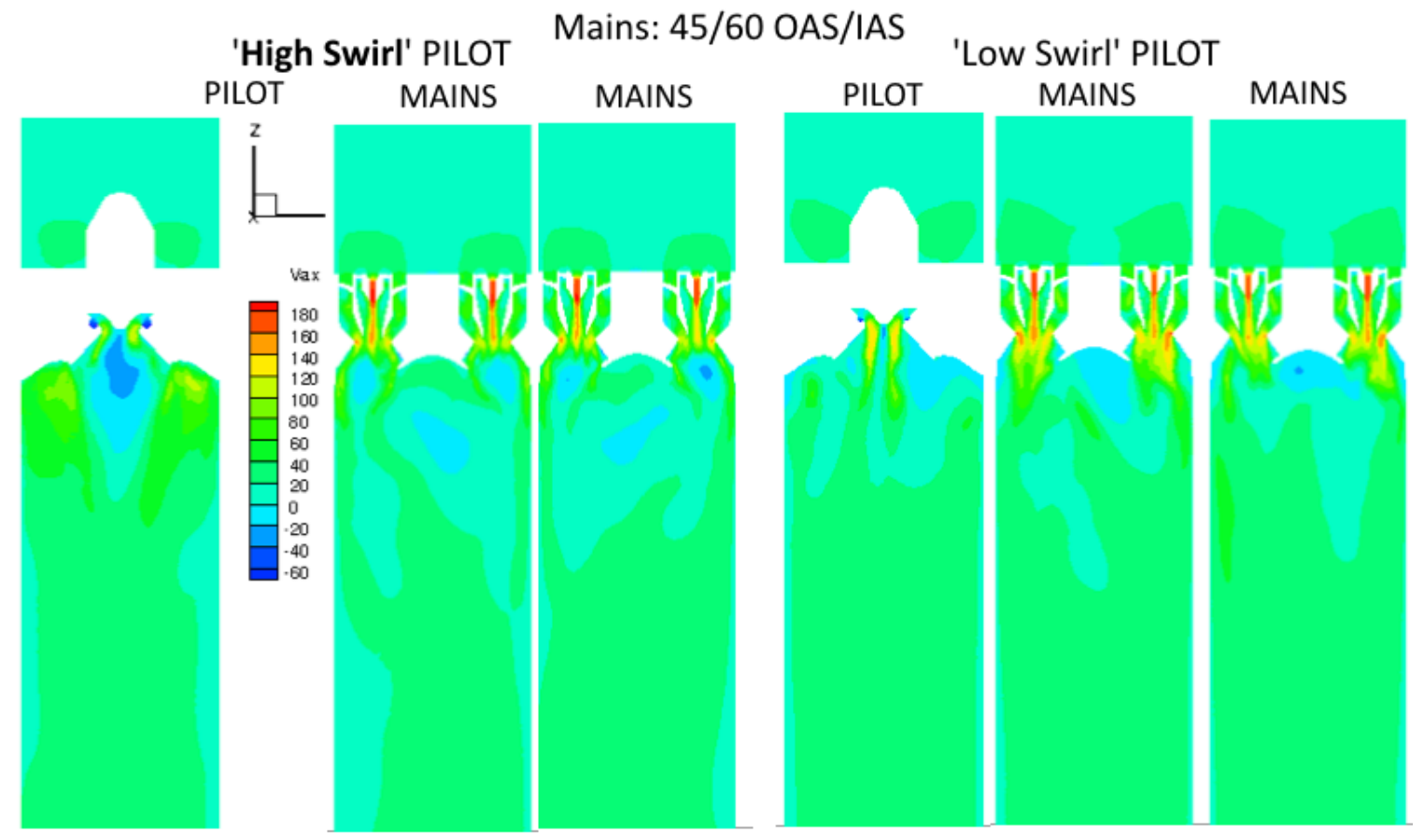

Figure 11. Axial velocity $(\mathrm{m} / \mathrm{s})$ contours in three axial cross-sections through centerline of two main injectors and the pilot injector. Left: 'High Swirl' Pilot Swirler. Right: 'Low Swirl' Pilot Swirler

\subsection{FIVE INJECTOR MODULE - REACTING FLOW COMPUTATIONS}

An optimized five-injector module that is a combination of a 'high-swirl' pilot swirler and $45^{\circ} / 60^{\circ}$ OAS/IAS main swirlers, was selected detailed reacting-flow analysis with NCC RANS and NCC TFNS/VLES. The flow conditions evaluated correspond to a 'medium' power engine cycle condition represented by $\mathrm{P}_{3}=130 \mathrm{psi}, 4 \% \Delta \mathrm{P}, \mathrm{T}_{3}=1000 \mathrm{~F}(811 \mathrm{~K})$ and $\mathrm{FAR}=0.0265$. The liquid fuel (Jet-A) is modeled with a lagrangian methodology, with initial conditions of the spray particles specified by measured particle data provided by Woodward FST. The fuel injector that feeds each main element was modeled by placing sixteen discrete fuel streams located circumferentially along the pre-filmer's annular surface, at an axial location immediately downstream of the pre-filmer's exit (see section 4.3 for more details). The pressureatomizing fuel injector that feeds the pilot element was modeled as a hollow-cone spray with a $60^{\circ}$ cone angle and $10^{\circ}$ cone-thickness angle, with an injection velocity of $30 \mathrm{~m} / \mathrm{s}$ and droplet SMD size of 8 microns. Due to the relatively small initial droplet SMD size of less than 10 microns, primary and/or secondary breakup models were not necessary in the current NCC computations.

The chemical reactions between the air and the vaporized Jet-A fuel were modeled with a reducedkinetics mechanism that was derived and tested for LDI2 analysis [Ref JPC $2014 \mathrm{Kundu}$ ]. The mechanism used for the current LDI3 analysis has been validated for NCC RANS against LDI2 experimental emissions data [Ref JPC 2014 Ajmani]. Some details of the Jet-A/Air mechanism are described in Appendix A. Turbulence closure is obtained by using a two-equation, cubic k- $\varepsilon$ model with variable C $\mu$ ([Shih 1998]) and dynamic wall functions with pressure gradient effects ([Shih 2000]). In the following sections, two sets of results are presented: (a) NCC RANS compared to NCC TFNS/VLES, both with laminar chemistry (i.e. no turbulence-chemistry interaction), and (b) NCC TFNS/VLES with a 'pdf-like' turbulence-chemistry interaction model compared to NCC TFNS/VLES with laminar chemistry.

\subsection{FIVE INJECTOR MODULE - REACTING FLOW PREDICTIONS (LAMINAR CHEMSITRY)}

A comparison of NCC RANS and NCC TFNS/VLES two-phase reacting flow computations with finite-rate reduced-kinetics Jet-A/Air modeling is described in this section. The current comparison uses laminar chemistry - the effects of turbulence-chemistry interaction will be described in the next section. 
Figures 12 and 13 show axial-velocity contours of the NCC results at various cross-sections through the five-injector module. A strong, prominent central recirculation zone (CRZ) behind the pilot is predicted by both of NCC RANS and NCC TFNS/VLES. The CRZ behind the four main injectors is slightly stronger, and better defined by NCC TFNS/VLES (figure 12, right). Figure 13 shows that NCC TFNS/VLES predicts a relatively symmetric and uniform flow pattern behind each of the four main injectors. The NCC RANS predictions show relatively large variations in the flow patterns for the four main injectors. This behavior is inconsistent with expectations, as the flow behind identically configured injectors should be largely identical, except for interaction effects from the central pilot injector.

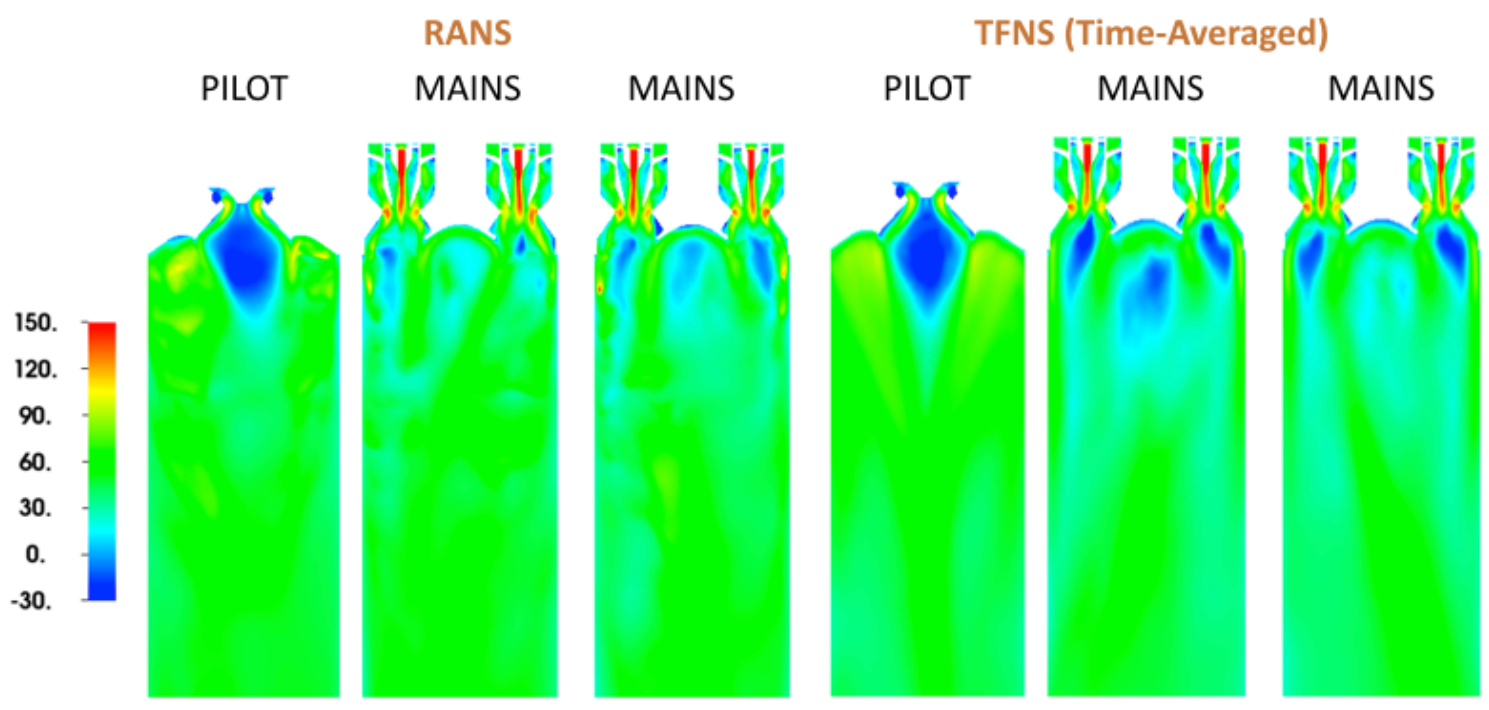

Figure 12. Axial velocity $(\mathrm{m} / \mathrm{s})$ contours in three axial cross-sections through centerline of two main injectors and the pilot injector. Left: RANS solution. Right: TFNS/VLES solution

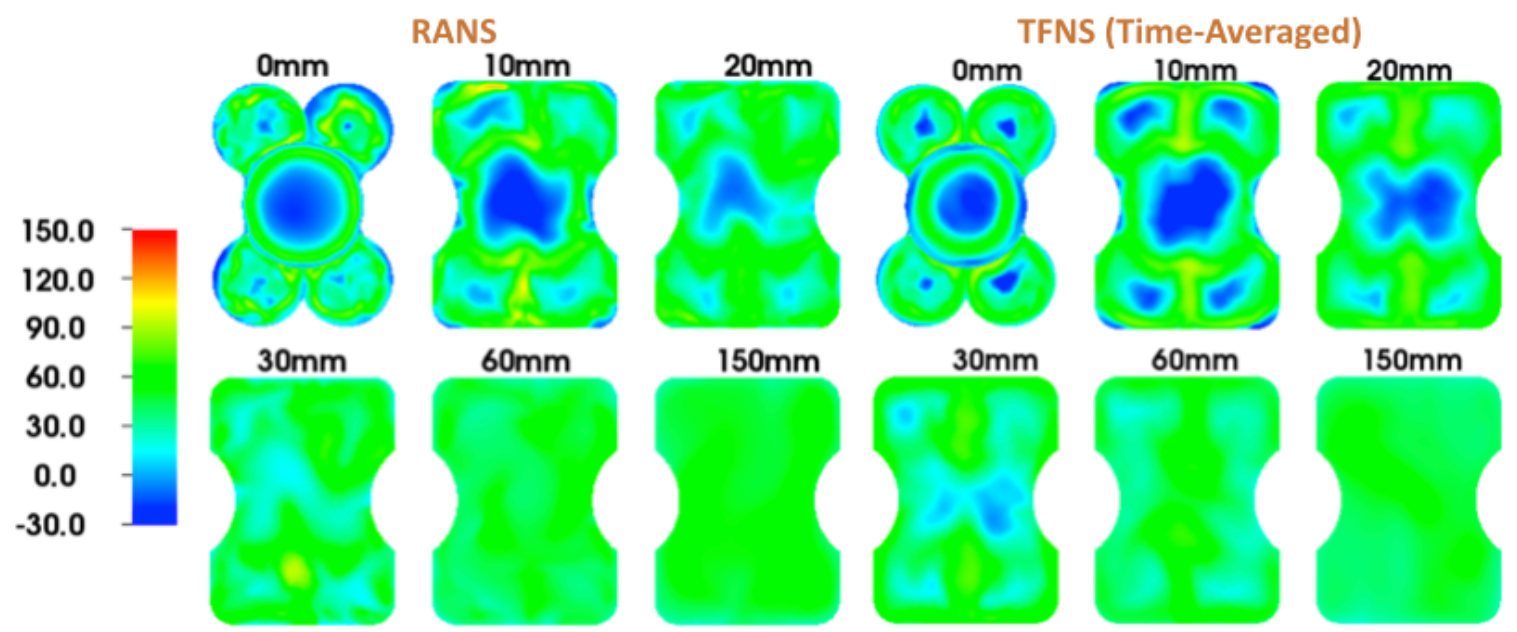

Figure 13. Axial velocity $(\mathrm{m} / \mathrm{s})$ contours in six cross-sections at various axial locations downstream of dump plane located at $0 \mathrm{~mm}$. Left: RANS solution. Right: TFNS/VLES solution

Figures 14 and 15 show temperature contours of the NCC results at various cross-sections through the five-injector module. The NCC RANS results (figure 14, left) show a very unstable flame with hot temperature streaks that are present near the combustor walls, at many locations axially downstream of the dump plane. The pilot injector creates a much shorter flame as compared to the main injectors. NCC TFNS/VLES predictions (figure 14, right) also indicate a very long flame region behind the main injectors, although the flame zones are much more uniform as compared to the RANS predictions. The presence of hot-streaks at the exit plane of the computation domain indicates that the NCC RANS flow is not 'fully- 
mixed' and has failed to attain mixture equilibrium. The TFNS/VLES contours show much better mixing as compared to NCC RANS, as seen at the $150 \mathrm{~mm}$ location comparison in figure 15 . The exit plane averaged temperatures (computed at the $150 \mathrm{~mm}$ plane) for NCC RANS and NCC TFNS/VLES are 1924K and $1684 \mathrm{~K}$, respectively. For reference, the equilibrium (fully mixed-out) temperature for the computed cycle condition is $1707 \mathrm{~K}$.

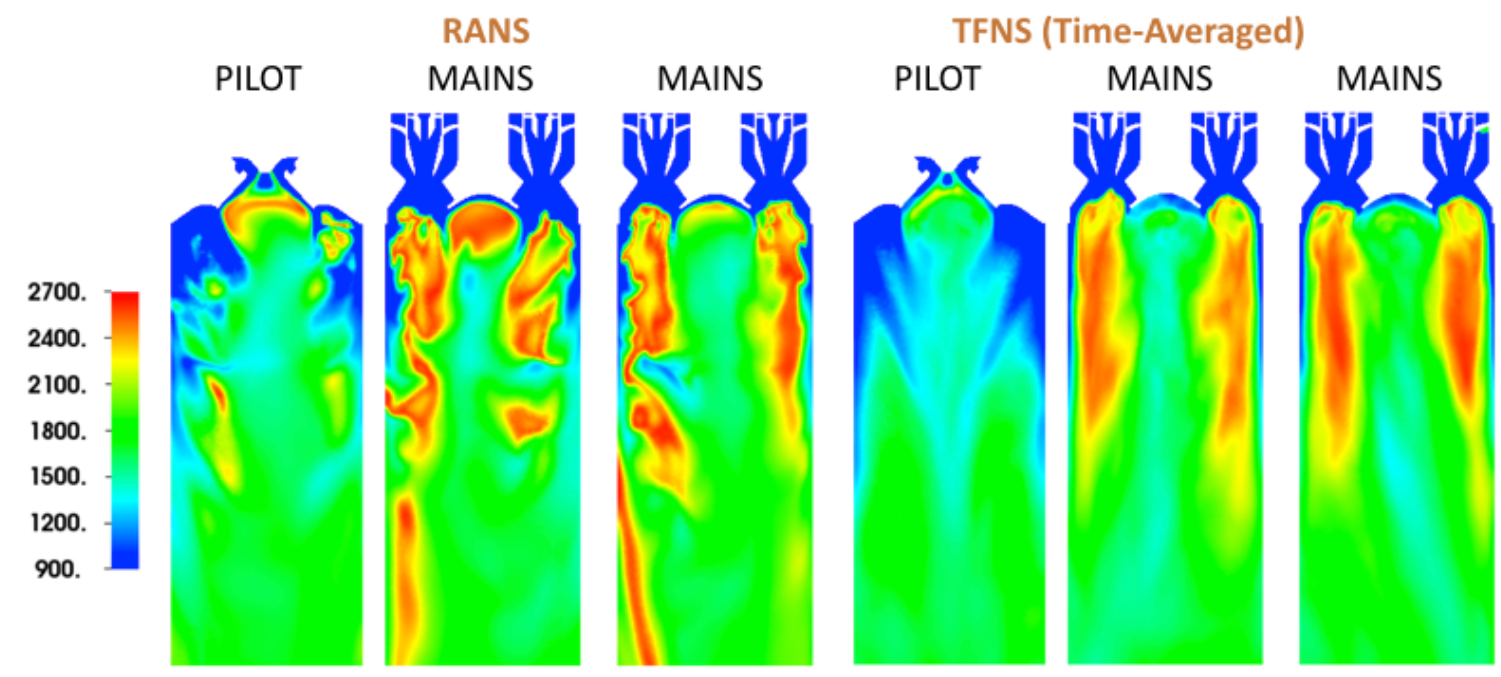

Figure 14. Temperature $(\mathrm{K})$ contours in three axial cross-sections through centerline of two main injectors and the pilot injector. Left: RANS solution. Right: TFNS/VLES solution

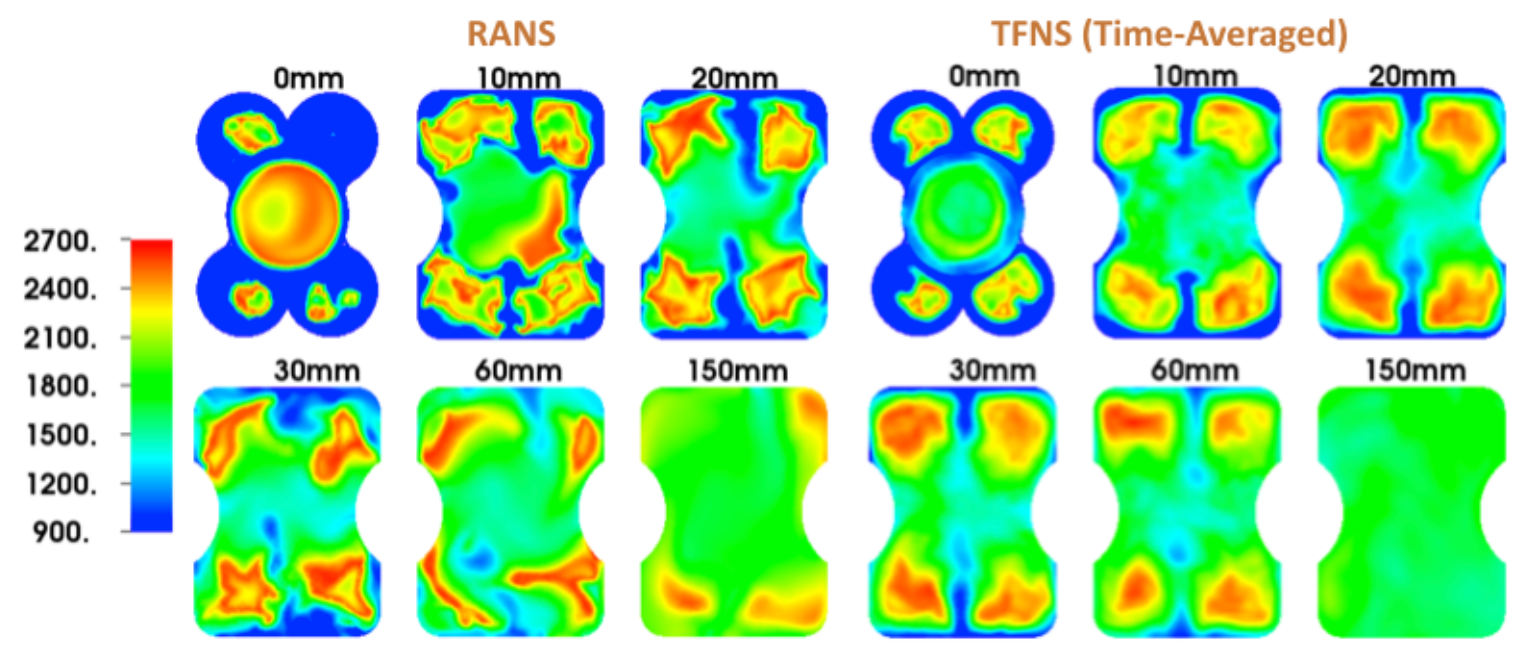

Figure 15. Temperature $(\mathrm{K})$ contours in six cross-sections at various axial locations downstream of dump plane located at $0 \mathrm{~mm}$. Left: RANS solution. Right: TFNS/VLES solution

The effect of hot temperature-streaks on predicted NOx emissions is shown in figures 16 and 17. The NCC RANS NOx predictions follow the irregularities of the hot zones (see figure 14, right), and large variations continue to exist at the exit plane of the computational domain (figure 17 left, $150 \mathrm{~mm}$ plane). The predictions with NCC TFNS/VLES are slightly better in that the variation of NOx at the exit plane (figure 17 right) is not as severe as that of NCC RANS. The computed EINOx ( $\mathrm{g}$ of NO/kg of FUEL) at the exit plane for NCC RANS is 25 , while that for NCC TFNS/VLES is a slightly higher value of 29. One explanation for the higher EINOx prediction of NCC TFNS/VLES is that the temperature field at the exit plane for TFNS/VLES is better mixed-out than that for RANS. Hence, the TFNS/VLES value for EINOx accounts for a much larger percentage of all of the possible NOx formation in the domain. The RANS EINOx value only has a partial accounting of all of the possible NOx formation, as can be seen by the 
pockets of high concentration NOx seen exiting the domain at the $150 \mathrm{~mm}$ plane. Extending the computational domain further downstream would allow for the RANS solution to be better mixed out, and allow for all of the NOx to be formed within the domain. This would result in a much greater predicted EINOx value with NCC RANS, than the value of 25 currently reported with the $150 \mathrm{~mm}$ long domain.

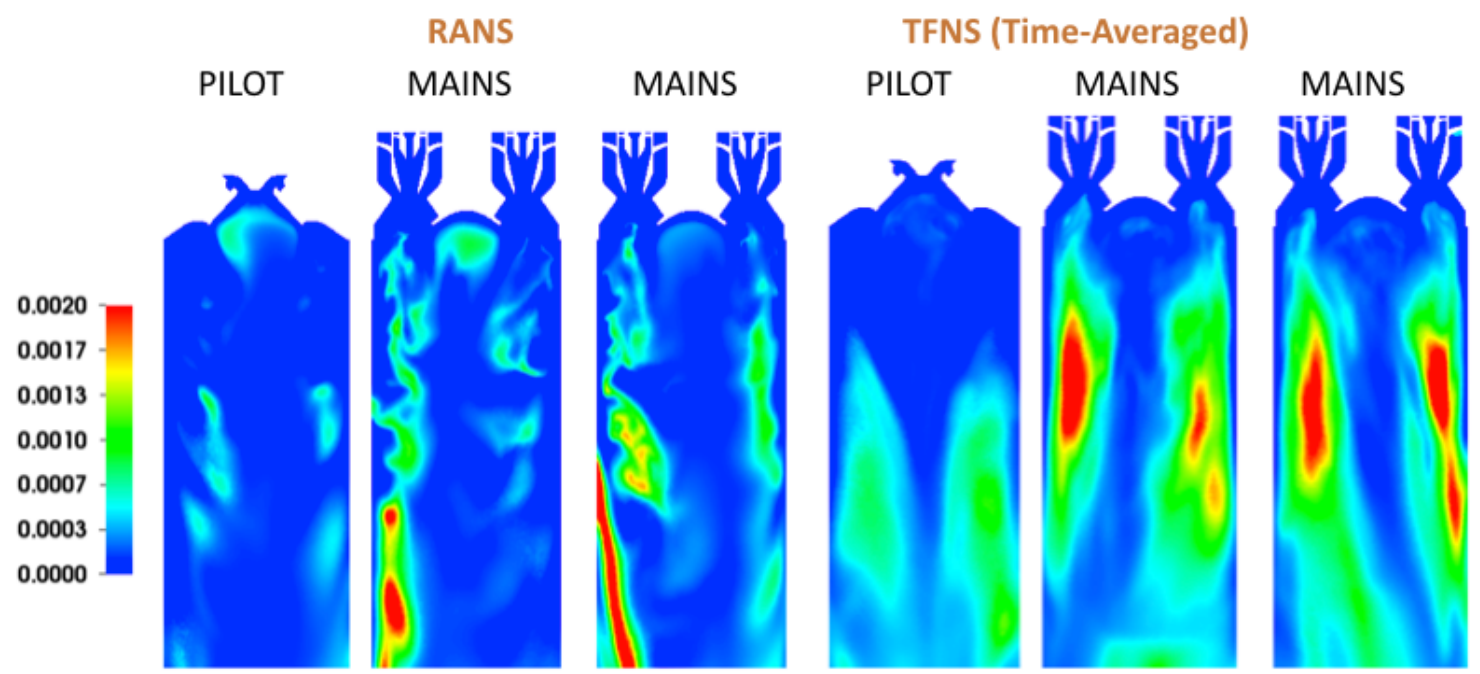

Figure 16. NO mass-fraction contours in three axial cross-sections through centerline of two main injectors and the pilot injector. Left: NCC RANS. Right: NCC TFNS/VLES

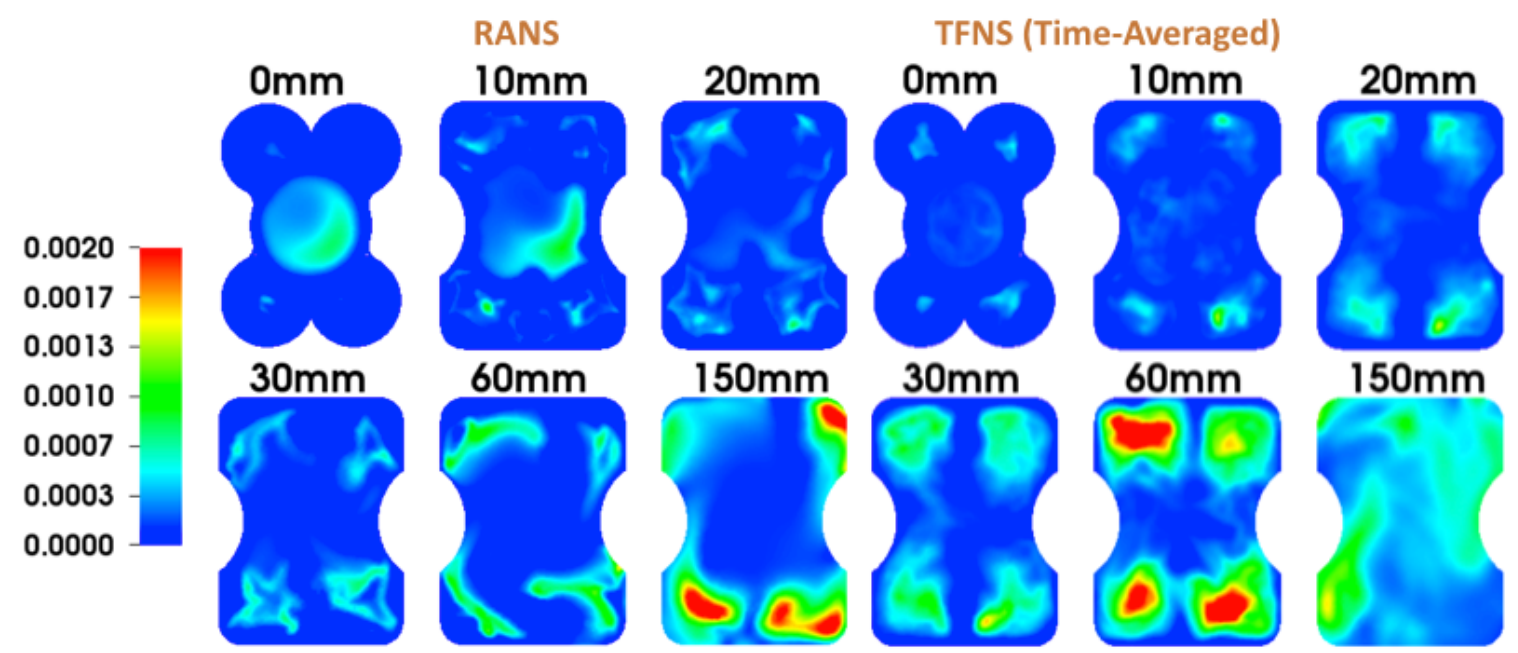

Figure 17. NO mass-fraction contours in six cross-sections at various axial locations downstream of dump plane located at $0 \mathrm{~mm}$. Left: NCC RANS. Right: NCC TFNS/VLES

\subsection{REACTING FLOW TFNS/VLES WITH SCALAR PDF-LIKE TURBULENCE CHEMISTRY INTERACTION MODEL}

A joint-scalar 'PDF-like' turbulence chemistry interaction model developed by [Ref Liu JPC 2015] was used with NCC TFNS/VLES to study its impact on the reacting flow-field of the five-element injector module described earlier. The TFNS/VLES solution obtained in section 5.3 was used as an initial condition to restart the computation with the 'PDF-like' solver. The PDF solver was coupled with TFNS/VLES at each physical time-step, and twenty-five Monte-Carlo particles per computational cell were used to model the turbulence chemistry interactions. A comparison of axial-velocity profiles (see figures 18 and 19) shows very marginal influence of the 'PDF-like' solver on the overall predicted aerodynamics of the five- 
element injector. This is to be expected, as the inclusion of turbulence chemistry interaction modeling would probably have the greatest impact on the temperature and species profiles of the injection module.

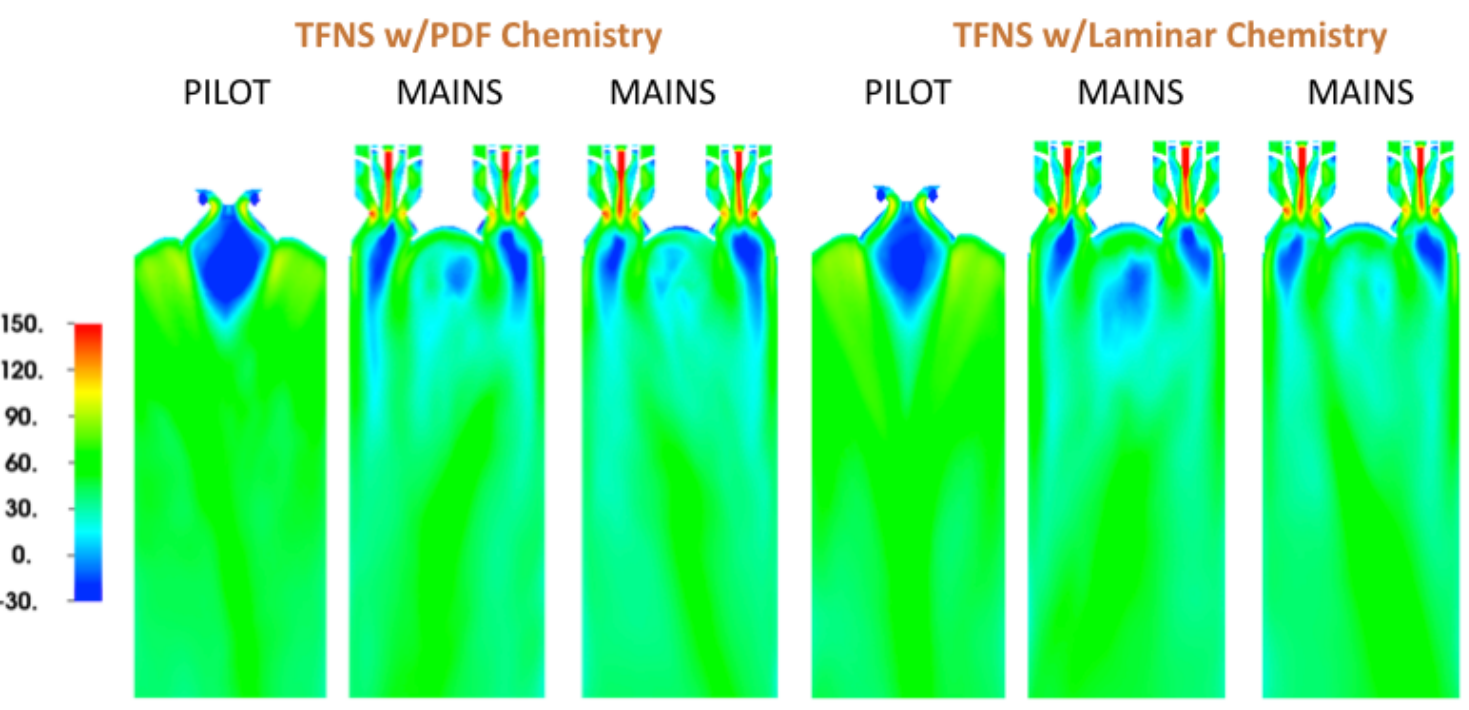

Figure 18. Axial velocity $(\mathrm{m} / \mathrm{s})$ contours in three axial cross-sections through centerline of two main injectors and the pilot injector. Left: TFNS w/PDF chemistry. Right: TFNS w/laminar chemistry

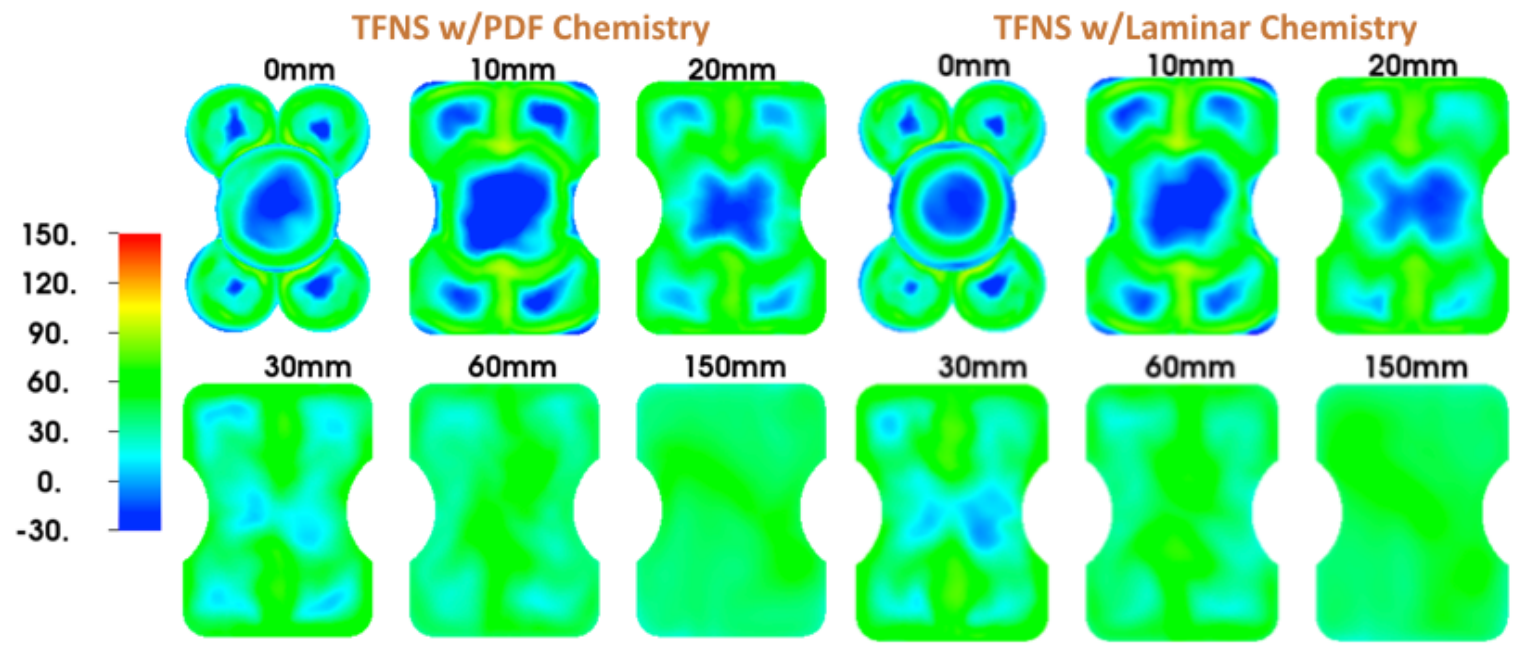

Figure 19. Axial velocity $(\mathrm{m} / \mathrm{s})$ contours in six cross-sections at various axial locations downstream of dump plane located at $0 \mathrm{~mm}$. Left: TFNS w/PDF chemistry. Right: TFNS w/laminar chemistry

The influence of the 'PDF-like' model on the temperature profile predictions with NCC TFNS/VLES is shown in figures 20 and 21 . The 'PDF-like' TFNS/VLES solution predicts much shorter flames behind the four main elements fueled by the pre-filming injector (see figure 20). In addition, the pilot injector has a low temperature zone that extends all the way from the $0 \mathrm{~mm}$ plane to the $60 \mathrm{~mm}$ plane (see figure 21). The exit plane averaged temperatures (computed at the $150 \mathrm{~mm}$ plane) for 'PDF-like' and 'laminar chemistry' solutions are $1650 \mathrm{~K}$ and $1684 \mathrm{~K}$, respectively. For reference, the equilibrium (fully mixed-out) temperature for the computed cycle condition is $1707 \mathrm{~K}$. These predictions are consistent with observations of [Ref Wey] that the 'PDF-like' model predicts 'cooler' flames as compared to 'laminar chemistry' flames. 


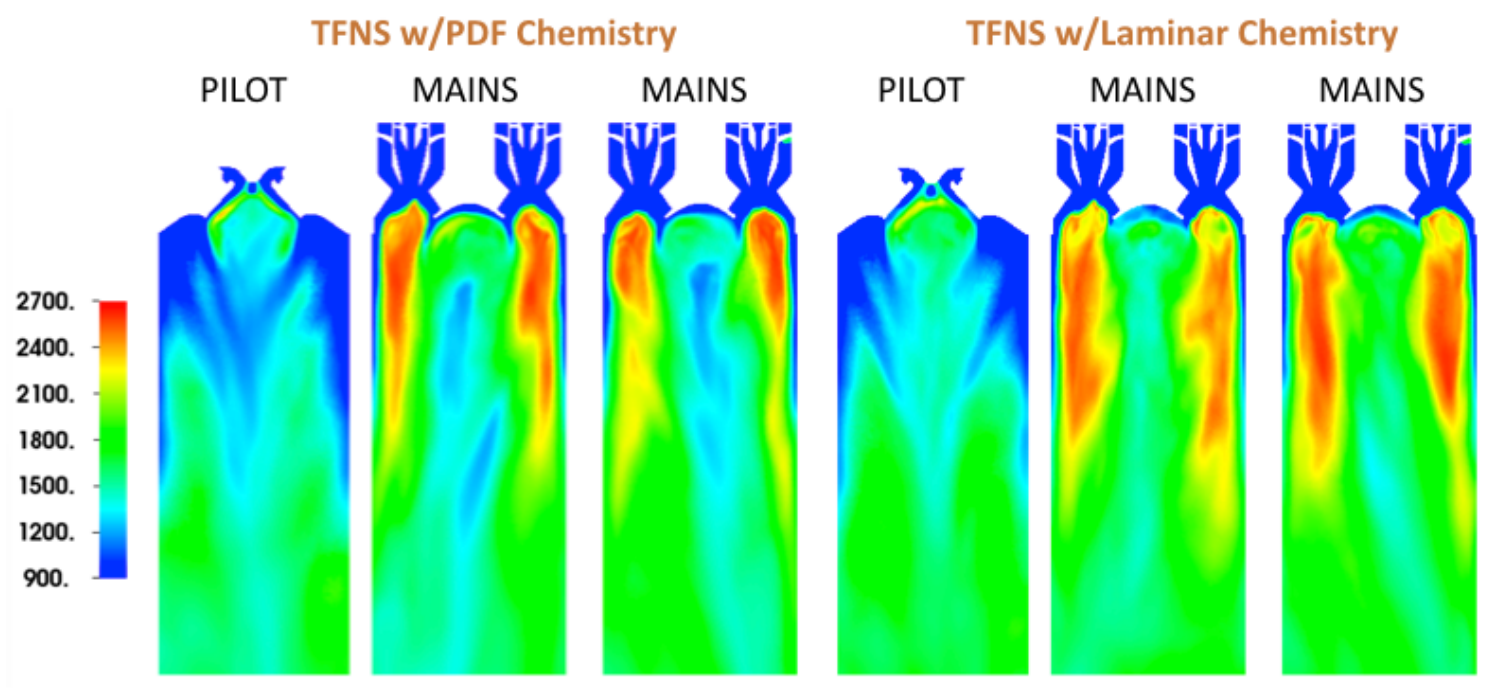

Figure 20. Temperature $(\mathrm{K})$ contours in three axial cross-sections through centerline of two main injectors and the pilot injector. Left: TFNS w/PDF chemistry. Right: TFNS w/laminar chemistry

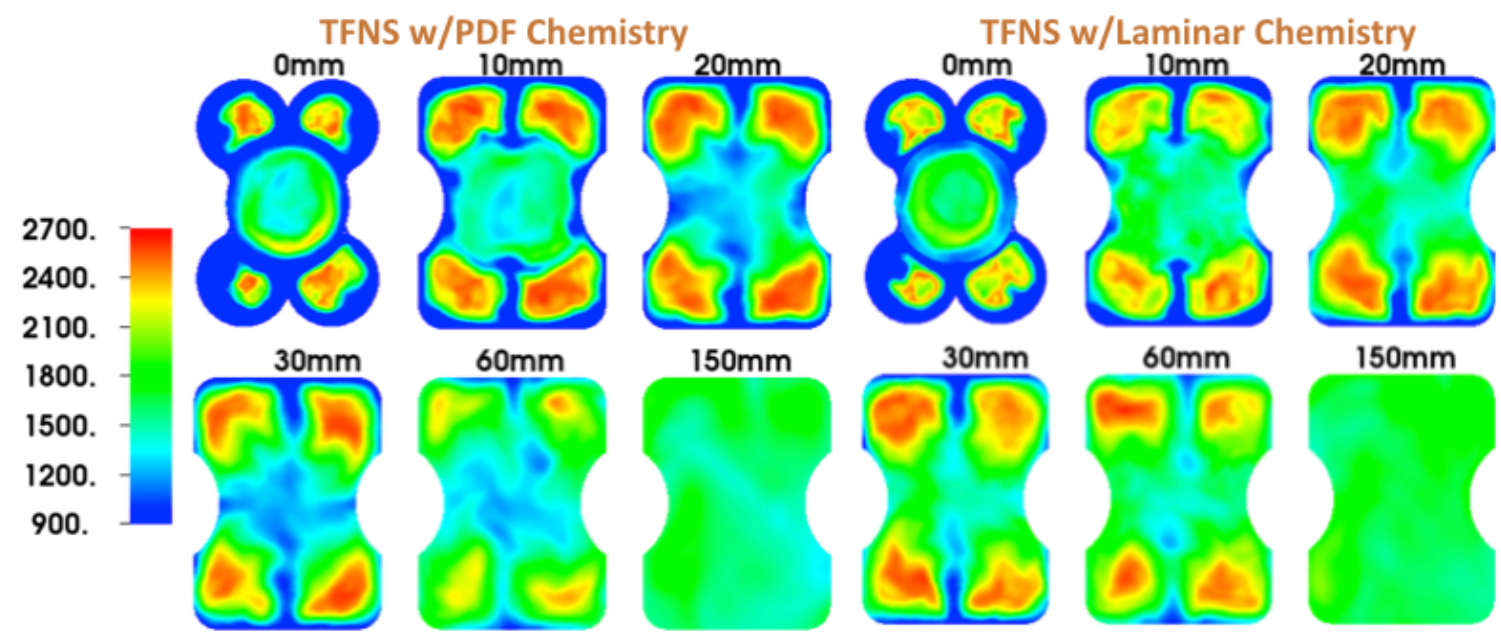

Figure 21. Temperature $(\mathrm{K})$ contours in six cross-sections at various axial locations downstream of dump plane located at $0 \mathrm{~mm}$. Left: TFNS w/PDF chemistry. Right: TFNS w/laminar chemistry

Figures 22 and 23 show the effect of coupling the 'PDF-like' solver with the TFNS/VLES solver on the NOx distribution within the injector. The 'PDF-like' solver, due to its prediction of lower temperature profiles and shorter flame zones, predicts much lower NOx than the 'laminar chemistry' approach. The overall EINOx prediction at the exit plane $(150 \mathrm{~mm})$, with the 'PDF-like' solver is 8.8 , which is more than a factor of 3 lower than the EINOx for the 'laminar chemistry' case (see section 5.3). A detailed comparison of the current EINOx prediction approach with NCC TFNS/VLES and 'PDF-like' modeling approach for the current LDI3 injector module will be done when experimental data becomes available. Meanwhile, further validation of this NCC modeling approach with the previous generation LDI2 injector modules, for which extensive experimental data is available [Ref Tacina JPC 2015], is recommended. 


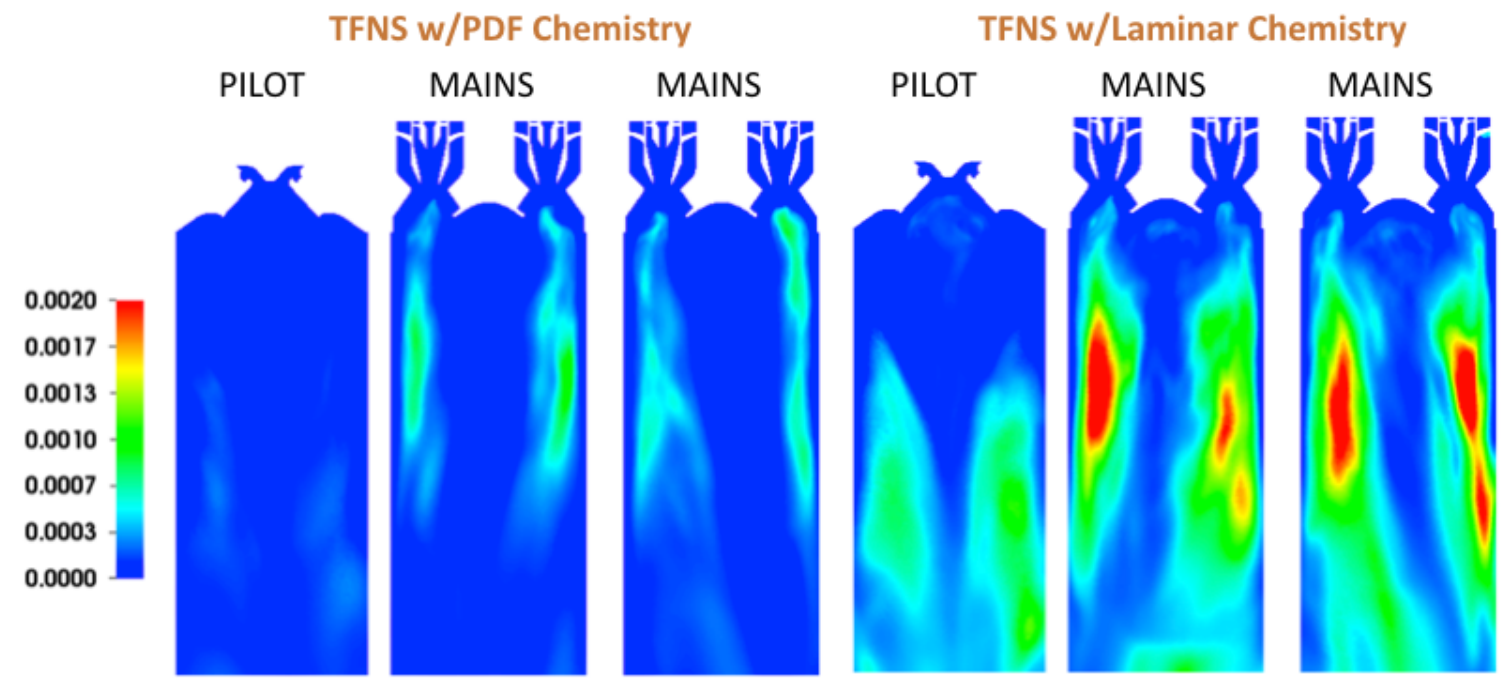

Figure 22. NO mass-fraction contours in three axial cross-sections through centerline of two main injectors and the pilot injector. Left: TFNS w/PDF chemistry. Right: TFNS w/laminar chemistry

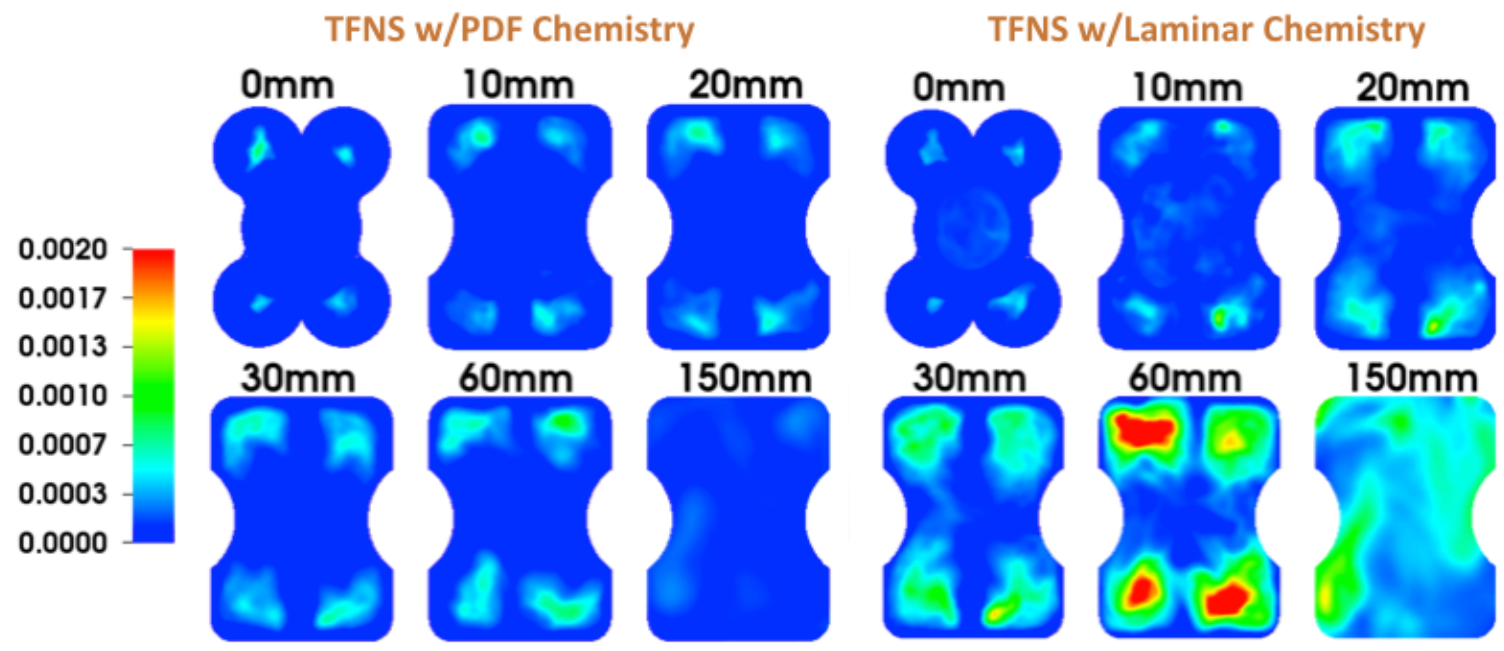

Figure 23. NO mass-fraction contours in six cross-sections at various axial locations downstream of dump plane located at $0 \mathrm{~mm}$. Left: TFNS w/PDF chemistry. Right: TFNS w/laminar chemistry

\subsection{SUMMARY AND CONCLUSIONS}

The National Combustor Code (NCC) was used to perform computational studies of a proposed LDI3 injector designed by Woodward, FST Inc. Non-. Geometry parametrics studied with the NCC included changing the turning angle and the orientation (counter or co-rotating) of the axial-bladed swirlers in the main injectors. The goal was to arrive at an optimized configuration that would meet the design requirements of maximum effective area, 'optimal' size of primary recirculation zone and minimal nearwall separation in the main injector venturi. The optimized single-element main-injector, fed by a newly designed pre-filming fuel injection scheme, was then analyzed with NCC for its effectiveness in providing acceptable fuel-air mixing, flame stability and temperature profiles. As a final analysis step, reacting flow simulations of an LDI-3 configuration consisting of four main-injectors (axial air flow, pre-filming fuel flow) and an integrated pilot injector (radial air flow, pressure-atomizing fuel flow) were performed. A finite-rate reduced-kinetics model and lagrangian-spray modeling (for the liquid phase droplets) were used. A comparison of reacting flow RANS, TFNS/VLES and TFNS/VLES with 'PDF-like' turbulencechemistry interaction indicates that the inclusion of 'PDF-like' flow physics in the computations creates a significant change in the temperature and emissions profile prediction of the LDI3 five-element injection 
module studied here. In order to verify the large impact of the 'PDF-like' model on the LDI3 NCC predictions, further validation with available experimental emissions data from LDI2 designs is highly recommended. NCC computations for an LDI3 seven-element injection module are in progress, and comparisons of emissions characteristics with experimental data will be made when testing at Woodward FST and NASA Glenn Research Center is completed. In addition, future work will include NCC computations for the full nineteen-element flame-tube configuration (two seven-element modules and one five-element module) that is currently undergoing experimental testing at NASA Glenn.

\subsection{REFERENCES}

[Ajmani 2013] Ajmani, K., Mongia, H.C. and Lee, P., “CFD Best Practices to Predict NOx, CO and Lean Blowout for Combustor Design," ASME IGTI Paper GT2013-95669, ASME Turbo Expo 2013, San Antonio TX, June 2013.

[Ajmani 2015] Ajmani, K., Mongia, H.C. and Lee, P., "Parametric Design of Injectors for LDI-3

Combustors,” AIAA Paper 2015-3785, 51st AIAA Joint Propulsion Conference, July 2015.

[Hicks 2007] Hicks, Y.R., Anderson, R.C., and Locke, R.J., “Optical Measurements in a Combustor Using a 9-Point Swirl-Venturi Fuel Injector,” ISABE 2007-1280.

[Hicks 2014] Hicks, Y.R., Tedder, S.A., Tacina, K.M., and Anderson, R.C., "Fundamental Study of a Single-Point Lean Direct Injector. Part II: A Comparison of Cold Flow and Burning Measurements," Paper 087IC-008, 2014 Technical Meeting of the Central States Section of the Combustion Institute, March 2014.

[Lee 2007] Lee, C.-M., Tacina, K.M. and Wey, C., "High Pressure Low NOx Emissions Research: Recent Progress at NASA Glenn Research Center,” ISABE 2007-1270.

[Lee 2013] Lee, C.-M., Chang, C., Kramer, S., and Hebron, J.T., "NASA Project develops next generation low-emission Combustor Technologies," 51st AIAA Aerospace Sciences Meeting, Dallas TX, AIAA Paper 2013-0540.

[Liu 2011] Liu, N.-S., Shih, T.-H. and Wey, C.T., "Numerical Simulations of Two-Phase Reacting Flow in a Single-Element Lean Direct Injection (LDI) Combustor Using NCC," NASA/TM-2011-217031.

[Mongia 2008] Mongia, H. C., 2008, "Recent Progress in Comprehensive Modeling of Gas Turbine Combustion," AIAA Paper 2008-1445.

[Shih 1998] Shih, T.-H., Chen, K.-H., Liu, N.-S., Lumley, J. L., "Modeling of Turbulent Swirling Flows," NASA-TM 1998-113112.

[Shih 2000] Shih, T.-H., Povinelli, L.A., Liu, N.-S and Chen, K.-H., "Generalized Wall Function for Complex Turbulent Flows," NASA TM 2000-209936.

[Suder 2013] Suder, K., Delaat, J., Hughes, C., Arend, D., and Celestina, M., "NASA ERA Project's Propulsion Technology Phase 1 Overview and Highlights of Accomplishments," 51st AIAA Aerospace Sciences Meeting, Dallas TX, AIAA Paper 2013-0414.

[Swanson 1997] Swanson, R.C., and Turkel, E., "Multistage Schemes With Multigrid for Euler and NavierStokes Equations," NASA TP-3631, 1997.

[Tacina 2005] Tacina, R., Lee, P., and Wey, C., “A Lean-Direct-Injection Combustor Using a 9 Point Swirl-Venturi Fuel Injector," ISABE 2005-1106.

[Tacina 2008] Tacina, K.M. and Wey, C., "NASA Glenn High Pressure Low NOx Emissions Research," NASA/TM-2008-214974. 